\title{
Assume-Guarantee Reasoning for Deadlock
}

Sagar Chaki, Software Engineering Institute

Nishant Sinha, Carnegie Mellon University

September 2006

TECHNICAL NOTE

CMU/SEI-2006-TN-028

Predictable Assembly from Certifiable Components Initiative Unlimited distribution subject to the copyright.

\section{CarnegieMellon}


This report was prepared for the

SEI Administrative Agent

ESC/XPK

5 Eglin Street

Hanscom AFB, MA 01731-2100

The ideas and findings in this report should not be construed as an official DoD position. It is published in the interest of scientific and technical information exchange.

This work is sponsored by the U.S. Department of Defense. The Software Engineering Institute is a federally funded research and development center sponsored by the U.S. Department of Defense.

Copyright 2006 Carnegie Mellon University.

NO WARRANTY

THIS CARNEGIE MELLON UNIVERSITY AND SOFTWARE ENGINEERING INSTITUTE MATERIAL IS FURNISHED ON AN "AS-IS" BASIS. CARNEGIE MELLON UNIVERSITY MAKES NO WARRANTIES OF ANY KIND, EITHER EXPRESSED OR IMPLIED, AS TO ANY MATTER INCLUDING, BUT NOT LIMITED TO, WARRANTY OF FITNESS FOR PURPOSE OR MERCHANTABILITY, EXCLUSIVITY, OR RESULTS OBTAINED FROM USE OF THE MATERIAL. CARNEGIE MELLON UNIVERSITY DOES NOT MAKE ANY WARRANTY OF ANY KIND WITH RESPECT TO FREEDOM FROM PATENT, TRADEMARK, OR COPYRIGHT INFRINGEMENT.

Use of any trademarks in this report is not intended in any way to infringe on the rights of the trademark holder.

Internal use. Permission to reproduce this document and to prepare derivative works from this document for internal use is granted, provided the copyright and "No Warranty" statements are included with all reproductions and derivative works.

External use. Requests for permission to reproduce this document or prepare derivative works of this document for external and commercial use should be addressed to the SEI Licensing Agent.

This work was created in the performance of Federal Government Contract Number FA8721-05-C0003 with Carnegie Mellon University for the operation of the Software Engineering Institute, a federally funded research and development center. The Government of the United States has a royalty-free government-purpose license to use, duplicate, or disclose the work, in whole or in part and in any manner, and to have or permit others to do so, for government purposes pursuant to the copyright license under the clause at 252.227-7013.

For information about purchasing paper copies of SEI reports, please visit the publications portion of our Web site (http://www.sei.cmu.edu/publications/pubweb.html 


\section{Table of Contents}

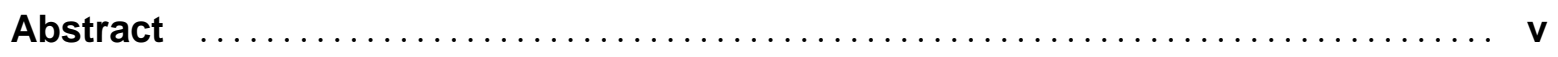

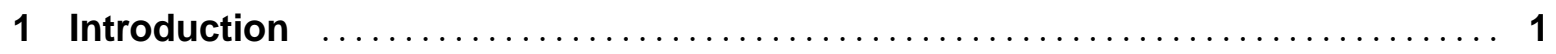

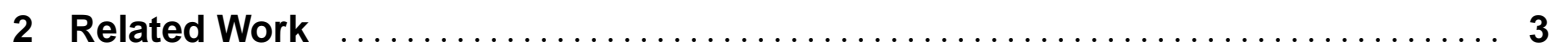

3 Failure Languages and Automata $\ldots \ldots \ldots \ldots \ldots \ldots \ldots \ldots \ldots \ldots \ldots$

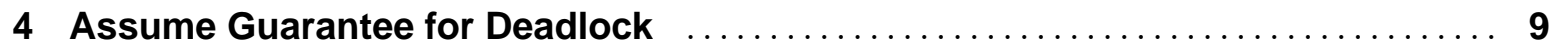

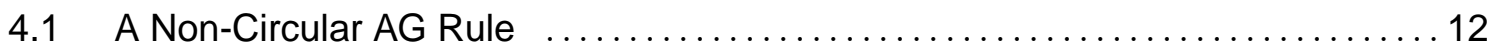

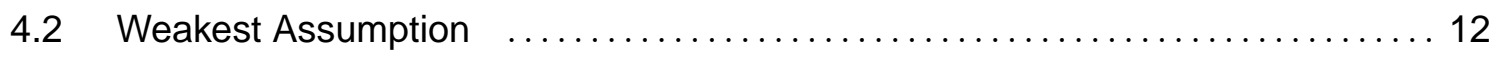

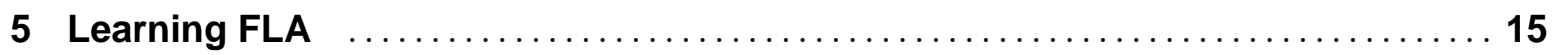

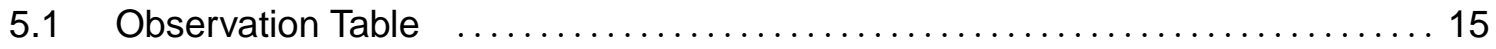

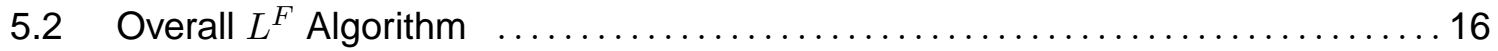

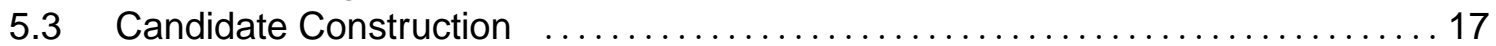

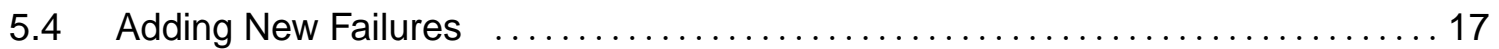

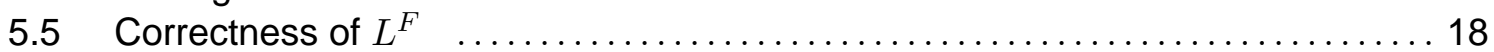

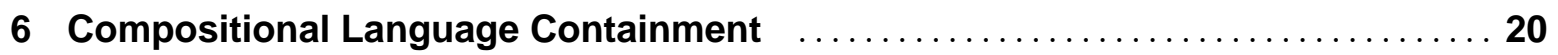

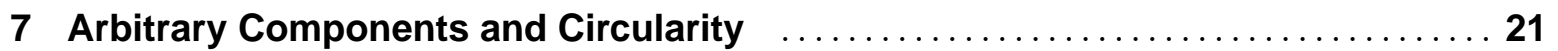

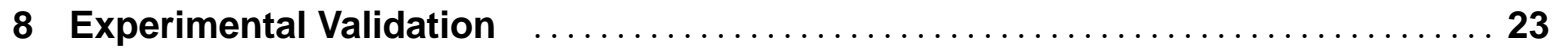

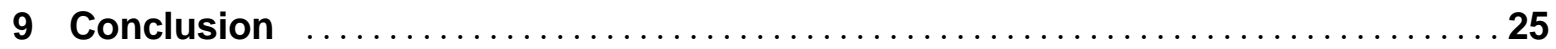

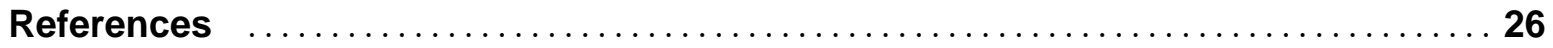




\section{List of Figures}

Figure 1: (a) LTS $M$ on $\Sigma=\{a, b, c\}$, (b) its FLA, and (c) its deterministic FLA. All

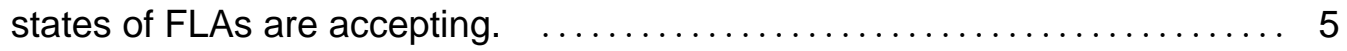

Figure 2: Algorithm MakeClosed. .................................. 16

Figure 3: Experimental results. $\mathrm{C}=\#$ of components; $\mathrm{St}=\#$ of states of largest component; $T=$ time (seconds); $M=$ memory (MB); $A=\#$ of states of largest assumption; ${ }^{*}$ = resource exhaustion; - = data unavailable; $\alpha=$ $1247 ; \beta=1708$. Best figures are shown in bold. 


\section{Abstract}

The use of learning to automate assume-guarantee style reasoning has received a lot of attention in recent years. This paradigm has already been used successfully for checking trace containment, as well as simulation between concurrent systems and their specifications. In this report, the learning-based automated assume-guarantee paradigm is extended to perform compositional deadlock detection. Failure automata is defined as a generalization of finite automata that accept regular failure sets. A learning algorithm $L^{F}$ is developed that constructs the minimal deterministic failure automaton accepting any unknown regular failure set using a minimally adequate teacher. This report shows how $L^{F}$ can be used for compositional regular failure language containment and deadlock detection, using non-circular and circular assume-guarantee rules. Finally, an implementation of techniques and encouraging experimental results on several nontrivial benchmarks are presented. 


\section{Introduction}

Ensuring deadlock freedom is one of the most critical requirements in the design and validation of systems. The biggest challenge toward the development of effective deadlock detection schemes remains the statespace explosion problem. Compositional reasoning [de Roever 98, McMillan 97, Grumberg 94] is recognized to be one of the most promising approaches for alleviating statespace explosion. This report presents an automated compositional deadlock detection procedure based on assume-guarantee (AG) [Pnueli 85] reasoning.

AG reasoning revolves around a proof rule that relates system components and assumptions about them to global system properties. Typically, to apply the proof rule, you need to construct manually appropriate assumptions that can discharge the premises of the rule. In most realistic situations, suitable assumptions are complicated. The absence of automated assumption-generation techniques has held back the wider practical adoption of AG reasoning.

An important breakthrough has been the use of learning algorithms for assumption construction [Cobleigh 03]. The general idea is to learn an automaton corresponding to the weakest assumption [Giannakopoulou 02] that can discharge the AG premises. The learning process is embedded in the overall verification procedure in a way that guarantees termination with the correct result. The choice of the learning algorithm is dictated by the kind of automaton that can represent the weakest assumption, which in turn depends on the verification goal. For example, in the case of trace containment [Cobleigh 03], the weakest assumptions are naturally represented as deterministic finite automata, and this leads to the use of the $L^{*}$ learning algorithm [Angluin 87]. Similarly, in the case of simulation [Chaki 05a], the corresponding choices are deterministic tree automata and the $L^{T}$ learning algorithm.

Nonetheless, neither learning algorithm is appropriate for deadlock detection. Word and tree automata are unable to capture failures [Hoare 85], a critical concept for understanding and detecting deadlocks. While you can transform any deadlock detection problem to an ordinary trace containment, such schemes invariably introduce new components and an exponential number of actions. As a result, these strategies are not scalable. Our work started with a search for an appropriate automata-theoretic formalism that can handle failures directly. Our deadlock detection algorithm uses learning-based automated AG reasoning and does not require additional actions or components.

Two key parts of our solution are: (1) a new type of acceptors for regular failure languages (RFLs) with a non-standard accepting condition and (2) a notion of parallel composition between these acceptors that is consistent with the parallel composition of the languages they accept. Our accepting condition is novel and employs a notion of maximality that crucially avoids introducing an exponential number of new actions. To the best of our knowledge, such acceptors and their composition have not been discussed before. In addition, we believe that this report presents the first use of learning in the context of automated AG reasoning for deadlock detection.

In Section 2, we present the theory of regular failure languages which are downward closed 
and define failure automata that exactly accept the set of regular failure languages. Although RFLs are closed under union and intersection but not under complementation, which is an acceptable tradeoff for the use of maximality. Further, we show a Myhill-Nerode-like theorem for RFLs and failure automata.

We show, in Section 3, that the failure language of a labeled transition system (LTS) $M$ is regular and checking deadlock freedom for $M$ is a particular instance of the problem of checking RFL containment. Then, we present an algorithm for checking containment of RFLs. We cannot check containment between failure languages $L_{1}$ and $L_{2}$ by complementing $L_{2}$ and intersecting with $L_{1}$, since (as we noted above) RFLs are not closed under complementation.

In Section 4, we present a sound and complete non-circular AG rule, AG-NC, on failure languages for checking failure language specifications. Given failure languages $L_{1}$ and $L_{S}$, we define the weakest assumption failure language $L_{W}$ : for every $L_{A}$ such that $L_{1} \| L_{A} \subseteq L_{S}$, $L_{A} \subseteq L_{W}$. We then show constructively that if failure languages $L_{1}$ and $L_{2}$ are regular, then $L_{W}$ uniquely exists, is regular, and is accepted by a minimum failure automaton $A_{W}$.

Section 5 details the development of an algorithm $L^{F}$ to learn the minimum deterministic failure automaton that accepts an unknown regular failure language $U$ using a minimally adequate teacher that can answer membership and candidate queries pertaining to $U$. We show how the teacher can be implemented using the RFL containment algorithm mentioned above.

In Section 6, we develop an automated and compositional deadlock detection algorithm that employs AG-NC and $L^{F}$.

Section 7 defines a circular AG proof rule AG-Circ for deadlock detection and shows how to use it for automated and compositional deadlock detection.

As we show in Section 8, we have implemented our approach in the ComFoRT [Chaki 05b] reasoning framework. We present encouraging results on several nontrivial benchmarks, including an embedded OS and Linux device drivers.

Finally, Section 9 summarizes our conclusions. 


\section{Related Work}

Machine-learning techniques have been used in several contexts related to verification [Peled 99, Groce 02, Alur 05a, Habermehl 05, Ernst 99]. We follow the approach of Cobleigh, Giannakopoulou, and Păsăreanu [Cobleigh 03] (respectively Chaki and colleagues [Chaki 05a]) to automate assume-guarantee reasoning for trace-containment (or simulation) between finite state systems. ${ }^{1}$ However, we apply this general paradigm for deadlock detection. This $L^{F}$ algorithm may also be of independent interest. Rivest and Schapire proposed an improvement to Angluin's $L^{*}$ that substantially improves its complexity [Rivest 93]. $L^{F}$ has the same spirit as this improved version of $L^{*}$. The use of circular AG rules was also investigated in the context of trace containment by Barringer, Giannakopoulou, and Păsăreanu [Barringer 03].

Overkamp explored the synthesis of supervisory controller for discrete-event systems [Overkamp 97] based on failure semantics [Hoare 85]. His notion of the least restrictive supervisor that guarantees deadlock-free behavior is similar to the weakest failure assumption in our case. However, our approach differs as follows: (1) We use failure automata to represent failure traces; (2) We use learning to compute the weakest failure assumption automatically; and (3) Our focus is on checking deadlocks in software modules. Williams, Thies and Ernst investigated an approach based on static analysis for detecting deadlocks that incorrect lock manipulation by Java programming language libraries can cause [Williams 05]. ${ }^{2}$ The problem of detecting deadlocks for pushdown programs communicating only via nested locking has been investigated by Kahlon, Ivancic and Gupta [Kahlon 05]. In contrast, we present a model-checking-based framework to compositionally verify deadlock freedom for non-recursive programs with arbitrary lock-based or rendezvous communication. Other non-compositional techniques for detecting deadlock have been investigated in context of partial-order reduction [Holzmann 03] and for checking refinement of CCS processes using a notion called stuck-free conformance that's more discriminative than failure trace refinement [Fournet 04].

Brookes and Roscoe use the failure model to show the absence of deadlock in undirectional networks [Brookes 91]. They also generalize the approach to the class of conflict-free networks via decomposition and local deadlock analysis. In contrast, we provide a completely automated framework for detecting deadlocks in arbitrary networks of asynchronous systems using rendezvous communication. Our formalism is based on an automata-theoretic representation of failure traces. Moreover, to analyze the deadlock freedom of a set of concurrent programs compositionally, we use both circular and non-circular assume-guarantee rules [Pnueli 85, de Roever 98, Barringer 03]. Amla and colleagues have presented a sound and complete assume-guarantee method in the context of an abstract process composition framework [Amla 03]. However, they do not discuss deadlock detection or explore the use of learning.

1 Alur, Madhusudan, and Nam have also investigated symbolic learning in this context [Alur 05b].

2 Williams, Thies and Ernst also also provide an excellent survey of related research [Williams 05]. 


\section{$3 \quad$ Failure Languages and Automata}

In this section, we present the theory of failure languages and failure automata. We consider a subclass of regular failure languages and provide a lemma relating regular failure languages (RFLs) and failure automata (FLA), analogous to Myhill-Nerode theorem for ordinary regular languages. We begin with a few standard definitions [Roscoe 97].

Definition 1 (Labeled Transition System) A labeled transition system (LTS) is a quadruple (S, Init, $\Sigma, \delta$ ) where: (i) $S$ is a set of states, (ii) Init $\subseteq S$ is a set of initial states, (iii) $\Sigma$ is a set of actions (alphabet), and (iv) $\delta \subseteq S \times \Sigma \times S$ is a transition relation.

We only consider LTSs such that both $S$ and $\Sigma$ are finite. We write $s \stackrel{\alpha}{\longrightarrow} s^{\prime}$ to mean $\left(s, \alpha, s^{\prime}\right) \in \delta$. A trace is any finite (possibly empty) sequence of actions, that is, the set of all traces is $\Sigma^{*}$. We denote an empty trace by $\epsilon$, a singleton trace $\langle\alpha\rangle$ by $\alpha$, and the concatenation of two traces $t_{1}$ and $t_{2}$ by $t_{1} \bullet t_{2}$. For any LTS $M=(S$, Init, $\Sigma, \delta)$, we define the function $\widehat{\delta}: 2^{S} \times \Sigma^{*} \rightarrow 2^{S}$ as follows:

$$
\widehat{\delta}(X, \epsilon)=X \quad \text { and } \quad \widehat{\delta}(\mathrm{X}, \mathrm{t} \bullet \alpha)=\left\{\mathrm{s}^{\prime} \mid \exists \mathrm{s} \in \widehat{\delta}(\mathrm{X}, \mathrm{t}) \cdot \mathrm{s} \stackrel{\alpha}{\longrightarrow} \mathrm{s}^{\prime}\right\}
$$

$M$ is said to be deterministic if $\mid$ Init $\mid=1$ and $\forall s \in S \cdot \forall \alpha \in \Sigma \cdot|\widehat{\delta}(\{s\}, \alpha)| \leq 1$ and complete if $\forall s \in S \cdot \forall \alpha \in \Sigma \cdot|\widehat{\delta}(\{s\}, \alpha)| \geq 1$. Thus if $M$ is both deterministic and complete then $\mid$ Init $\mid=1$ and $\forall s \in S . \forall t \in \Sigma^{*} \cdot|\widehat{\delta}(\{s\}, t)|=1$. In this case, we write $\widehat{\delta}(s, t)$ to mean the only element of $\widehat{\delta}(\{s\}, t)$.

Definition 2 (Finite Automaton) A finite automaton is a pair $(M, F)$ such that $M=(S$, Init, $\Sigma, \delta)$ is an LTS and $F \subseteq S$ is a set of final states.

Let $G=(M, F)$ be a finite automaton. Then, $G$ is said to be deterministic (complete) iff the underlying LTS $M$ is deterministic (complete).

Definition 3 (Refusal) Let $M=(S$, Init, $\Sigma, \delta)$ be an LTS and $s \in S$ be any state of $M$. We say that $s$ refuses an action $\alpha$ iff $\forall s^{\prime} \in S \cdot\left(s, \alpha, s^{\prime}\right) \notin \delta$. We say that $s$ refuses a set of actions $R$ and denote this by $\operatorname{Re} f(s, R)$, iff $s$ refuses every element of $R$. Note that the following holds: (i) $\forall s$. Ref( $(s, \emptyset)$ and (ii) $\forall s, R, R^{\prime}$. Ref $(s, R) \wedge R^{\prime} \subseteq R \Longrightarrow \operatorname{Ref}\left(s, R^{\prime}\right)$ (i.e., refusals are downward-closed).

Definition 4 (Failure) Let $M=(S$, Init, $\Sigma, \delta)$ be an LTS. A pair $(t, R) \in \Sigma^{*} \times 2^{\Sigma}$ is said to be a failure of $M$ iff there exists some $s \in \widehat{\delta}($ Init, $t)$ such that $\operatorname{Re} f(s, R)$. The set of all failures of an LTS $M$ is denoted by $\mathcal{F}(M)$.

Note that a failure consists of both a trace and a refusal set. A (possibly infinite) set of failures $L$ is said to be a failure language. Let us denote $2^{\Sigma}$ by $\widehat{\Sigma}$. Note that $L \subseteq \Sigma^{*} \times \widehat{\Sigma}$. 


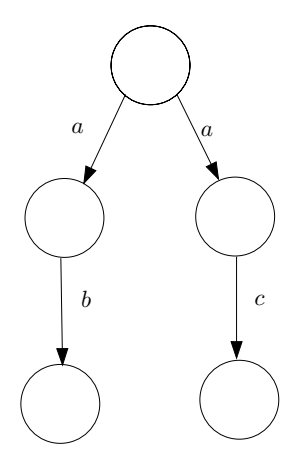

(a)

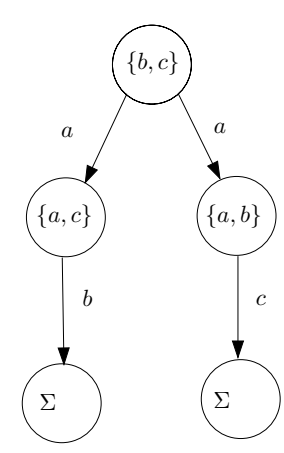

(b)

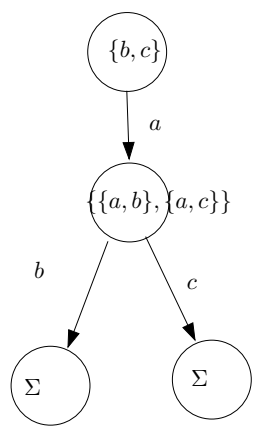

(c)

Figure 1: (a) LTS $M$ on $\Sigma=\{a, b, c\}$, (b) its FLA, and (c) its deterministic FLA. All states of FLAs are accepting.

The union and intersection of failure languages are defined in the usual way. The complement of $L$, denoted by $\bar{L}$, is defined to be $\left(\Sigma^{*} \times \widehat{\Sigma}\right) \backslash L$. A failure language is said to be downward-closed iff the following holds:

$$
\forall t \in \Sigma^{*} \cdot \forall R \in \widehat{\Sigma} \cdot(t, R) \in L \Longrightarrow \forall R^{\prime} \subseteq R .\left(t, R^{\prime}\right) \in L
$$

In general, failure languages may not be downward closed; however, we will show later that failure languages generated from LTSs are always downward closed because the refusal sets at each state of an LTS are downward closed. In this report, we focus on downward-closed failure languages, in particular, regular failure languages.

Definition 5 (Deadlock) An LTS $M$ is said to deadlock iff $\mathcal{F}(M) \cap\left(\Sigma^{*} \times\{\Sigma\}\right) \neq \emptyset$. In other words, $M$ deadlocks iff it has a reachable state that refuses every action in its alphabet.

Let us denote the failure language $\Sigma^{*} \times\{\Sigma\}$ by $L_{D l k}$. Then, it follows that $M$ is deadlock free iff $\mathcal{F}(M) \subseteq \overline{L_{D l k}}$.

Maximality. Let $P$ be any subset of $\widehat{\Sigma}$. The set of maximal elements of $P$ is denoted by $\operatorname{Max}(P)$ and defined as follows: $\operatorname{Max}(P)=\left\{R \in P \mid \forall R^{\prime} \in P . R \not \subset R^{\prime}\right\}$.

For example, if $P=\{\{a\},\{b\},\{a, b\},\{a, c\}\}$, then $\operatorname{Max}(P)=\{\{a, b\},\{a, c\}\}$. A subset $P$ of $\widehat{\Sigma}$ is said to be maximal iff it is non-empty and $\operatorname{Max}(P)=P$. Intuitively, failure automata are finite automata whose final states are labeled with maximal refusal sets. Thus, a failure $(t, R)$ is accepted by a failure automaton $M$ iff upon receiving input $t, M$ reaches a final state labeled with a refusal $R^{\prime}$ such that $R \subseteq R^{\prime}$. With the concept of maximality, we use only the upper bounds of a set (according to subset partial order) to represent the complete set and thereby concisely represent downward-closed failed languages.

Definition 6 (Failure Automaton) A failure automaton ( $F L A)$ is a triple $(M, F, \mu)$ such that $M=(S$, Init, $\Sigma, \delta)$ is an $L T S, F \subseteq S$ is a set of final states, and $\mu: F \rightarrow 2^{\widehat{\Sigma}}$ is a mapping from the final states to $2^{\widehat{\Sigma}}$ such that: $\forall s \in F \cdot \mu(s) \neq \emptyset \wedge \mu(s)=\operatorname{Max}(\mu(s))$. 
Let $A=(M, F, \mu)$ be a FLA. Then $A$ is said to be deterministic (respectively complete) iff the underlying LTS $M$ is deterministic (respectively complete). Part (a) of Figure 1 shows an LTS over $\Sigma=\{a, b, c\}$. Parts (b) and (c) show the corresponding FLA and its deterministic version, respectively.

Definition 7 (Language of an FLA) Let $A=(M, F, \mu)$ be an FLA such that $M=(S$, Init $, \Sigma, \delta)$. Then, a failure $(t, R)$ is accepted by $A$ iff the following holds:

$$
\exists s \in F \cdot \exists R^{\prime} \in \mu(s) . s \in \widehat{\delta}(\text { Init }, t) \wedge R \subseteq R^{\prime}
$$

The language of $A$, denoted by $\mathcal{L}(A)$, is the set of all failures accepted by $A$.

Every deterministic FLA (DFLA) $A$ can be extended to a complete DFLA $A^{\prime}$ such that $\mathcal{L}\left(A^{\prime}\right)=\mathcal{L}(A)$ by adding a non-final sink state. In the rest of this report, we consider FLA and languages over a fixed alphabet $\Sigma .^{3}$

Lemma 1 A language is accepted by an FLA iff it is accepted by a deterministic FLA, that is, deterministic FLA have the same accepting power as FLA in general.

Proof. By subset construction. Let $L$ be a language accepted by some FLA $A=(M, F, \mu)$. We construct a deterministic FLA $A^{\prime}=\left(M^{\prime}, F^{\prime}, \mu^{\prime}\right)$ as follows: The deterministic finite automaton $G^{\prime}=\left(M^{\prime}, F^{\prime}\right)$ is obtained by the standard subset construction from the finite automaton $G=(M, F)$. For any state $s^{\prime}$ of $M^{\prime}$, let us denote $\Psi\left(s^{\prime}\right)$ as the set of states of $M$ from which $s^{\prime}$ was derived by the subset construction. To define $\mu^{\prime}$, consider any final state $s^{\prime} \in F^{\prime}$. We know that $\Psi\left(s^{\prime}\right) \cap F \neq \emptyset$. Let $P=\bigcup_{s \in \Psi\left(s^{\prime}\right) \cap F} \mu(s)$. Then $\mu^{\prime}\left(s^{\prime}\right)=\operatorname{Max}(P)$.

Let Init and Init' be the initial states of $M$ and $M^{\prime}$, respectively. Now, to show that $\mathcal{L}\left(A^{\prime}\right)=L$, consider any failure $(t, R)$. Then

$$
\begin{gathered}
(t, R) \in \mathcal{L}\left(A^{\prime}\right) \Longleftrightarrow \exists s^{\prime} \in \widehat{\delta}\left(\text { Init }^{\prime}, t\right) \cap F^{\prime} \cdot \exists R^{\prime} \in \mu^{\prime}\left(s^{\prime}\right) \cdot R \subseteq R^{\prime} \\
\Longleftrightarrow \exists s^{\prime} \in \widehat{\delta}\left(\text { Init }^{\prime}, t\right) \cap F^{\prime} \cdot \exists s \in \Psi\left(s^{\prime}\right) \cap F \cdot \exists R^{\prime} \in \mu(s) \cdot R \subseteq R^{\prime} \\
\Longleftrightarrow \exists s \in \widehat{\delta}(\text { Init }, t) \cap F \cdot \exists R^{\prime} \in \mu(s) \cdot R \subseteq R^{\prime} \Longleftrightarrow(t, R) \in \mathcal{L}(A)=L
\end{gathered}
$$

Regular Failure Languages (RFLs). A failure language is said to be regular iff it is accepted by some FLA. It follows from the definition of FLAs that RFLs are downward closed. Hence, the set of RFLs is closed under union and intersection but not under complementation. ${ }^{4}$ In addition, every RFL is accepted by an unique minimal deterministic FLA. The following Lemma is analogous to the Myhill-Nerode theorem for regular languages and ordinary finite automata.

3 FLA are closely related to automata on guarded strings [Kozen 01], which contain arbitrary transition labels drawn from a partially ordered set. In contrast, the state labels (refusals) in FLA are only maximal elements from such a set. Further, since it suffices to consider refusals at the end of a trace for checking deadlock freedom, we only label the final states of an FLA.

4 For example, consider $\Sigma=\{\alpha\}$ and the RFL $L=\Sigma^{*} \times\{\emptyset\}$. Then $\bar{L}=\Sigma^{*} \times\{\{\alpha\}\}$ is not downward-closed and hence is not an RFL. 
Lemma 2 Every regular failure language(RFL) is accepted by an unique (up to isomorphism) minimal deterministic finite failure automaton.

Proof. Our proof follows that of the Myhill-Nerode theorem for finite automata. Let $L$ be any RFL. Let us define an equivalence relation $\equiv$ over $\Sigma^{*}$ as follows:

$$
u \equiv v \Longleftrightarrow \forall(t, R) \in \Sigma^{*} \times \widehat{\Sigma} \cdot(u \bullet t, R) \in L \Longleftrightarrow(v \bullet t, R) \in L
$$

For any $u \in \Sigma^{*}$, we denote the equivalence class of $u$ by $[u]$. Let us define a finite automaton $G=(M, F)$ where $M=(S$, Init, $\Sigma, \delta)$ such that: (i) $S=\left\{[u] \mid u \in \Sigma^{*}\right\}$, (ii) Init $=\{[\epsilon]\}$, (iii) $\forall u \in \Sigma^{*} \cdot \forall \alpha \in \Sigma \cdot[u] \stackrel{\alpha}{\longrightarrow}[u \bullet \alpha]$, and (iv) $F=\{[u] \mid \exists R \in \widehat{\Sigma} \cdot(u, R) \in L\}$.

Also, let us define a function $\mu$ as follows: Consider any $[u] \in F$ and let $P \subseteq \widehat{\Sigma}$ be defined as $P=\{R \mid \exists v \cdot v \equiv u \wedge(v, R) \in L\}$. Note that since $[u] \in F, P \neq \emptyset$. Then $\mu([u])=\operatorname{Max}(P)$. Let $A$ be the FLA $(M, F, \mu)$.

We first show by contradiction that $A$ is deterministic. First, note that $\mid$ Init $\mid=1$. Next, suppose that $A$ is nondeterministic. Then there exists two traces $u \in \Sigma^{*}$ and $v \in \Sigma^{*}$ and an action $\alpha \in \Sigma$ such that $u \equiv v$ but $u \bullet \alpha \not \equiv v \bullet \alpha$. Then there exists a failure $(t, R)$ such that $(u \bullet \alpha \bullet t, R) \in L \Longleftrightarrow(v \bullet \alpha \bullet t, R) \notin L$. But then there exists a failure $\left(t^{\prime}, R\right)=(\alpha \bullet t, R)$ such that $\left(u \bullet t^{\prime}, R\right) \in L \Longleftrightarrow\left(v \bullet t^{\prime}, R\right) \notin L$. This implies that $u \neq \equiv v$ which is a contradiction.

Next, we show that: (C1) for any trace $t, \widehat{\delta}($ Init,$t)=[t]$. The proof proceeds by induction on the length of $t$. For the base case, suppose $t=\epsilon$. Then $\widehat{\delta}($ Init, $t)=$ Init $=[\epsilon]$. Now suppose $t=t^{\prime} \bullet \alpha$ for some trace $t^{\prime}$ and action $\alpha$. By the inductive hypothesis, $\widehat{\delta}\left(\right.$ Init,$\left.t^{\prime}\right)=\left[t^{\prime}\right]$. Also, from the definition of $A$, we know that $\left[t^{\prime}\right] \stackrel{\alpha}{\longrightarrow}\left[t^{\prime} \bullet \alpha\right]$. Hence, $\widehat{\delta}($ Init,$t)=\widehat{\delta}\left(\right.$ Init,$\left.t^{\prime} \bullet \alpha\right)=\left[t^{\prime} \bullet \alpha\right]=[t]$. This completes the proof.

Now consider any DFLA $A^{\prime}=\left(M^{\prime}, F^{\prime}, \mu^{\prime}\right)$ where $M^{\prime}=\left(S^{\prime}\right.$, Init $\left.^{\prime}, \Sigma, \delta^{\prime}\right)$ such that $\mathcal{L}\left(A^{\prime}\right)=L$. Let us define a function $\Omega: S^{\prime} \rightarrow S$ as follows: $\forall t \in \Sigma^{*} . \Omega\left(\widehat{\delta}\left(\right.\right.$ Init $\left.\left.^{\prime}, t\right)\right)=\widehat{\delta}($ Init,$t)$. First we show that $\Omega$ is well-defined. Consider any two traces $u$ and $v$ such that $\widehat{\delta}\left(\right.$ Init $\left.^{\prime}, u\right)=\widehat{\delta}\left(\right.$ Init $\left.^{\prime}, v\right)$. Then for any failure $(t, R), A^{\prime}$ accepts $(u \bullet t, R)$ iff it also accepts $(v \bullet t, R)$. Since $A^{\prime}$ accepts $L$, we find that $u \equiv v$. Combining this equality with $\mathbf{C} 1$ above we have $\widehat{\delta}($ Init,$u)=[u]=[v]=\widehat{\delta}($ Init,$v)$. Therefore, $\widehat{\delta}($ Init,$u)=\widehat{\delta}($ Init,$v)$ which proves that $\Omega$ is well-defined. In addition, $\Omega$ is a surjection since for any state $[u]$ of $A$ we have the following from $\mathbf{C 1}$ above: $[u]=\widehat{\delta}($ Init,$u)=\Omega\left(\widehat{\delta}\left(\right.\right.$ Init $\left.\left.^{\prime}, u\right)\right)$.

We are now ready to prove the main result. In essence, we show that $A$ is the unique minimal DFLA that accepts $L$. We have already shown that $A$ is deterministic. To show that $\mathcal{L}(A)=L$ we observe that for any trace $t$ and any refusal $R$, the following holds:

$$
(t, R) \in L \Longleftrightarrow[t] \in F \wedge \exists R^{\prime} \in \mu([t]) \cdot R \subseteq R^{\prime} \Longleftrightarrow(t, R) \in \mathcal{L}(A)
$$

Next, recall that $\Omega$ defined above is a surjection. Hence, $A^{\prime}$ must have at least as many states as $A$. Since $A^{\prime}$ is an arbitrary DFLA accepting $L, A$ must be a minimal DFLA that accepts $L$. To show that $A$ is unique up to isomorphism, let $A^{\prime}$ be another minimal DFLA accepting $L$. In this case, $\Omega$ must be a bijection. We show that $\Omega$ is also an isomorphism. 
Let us write $\Omega^{-1}$ to mean the inverse of $\Omega$. Note that $\Omega^{-1}$ is also a bijection, and more specifically, $\forall t \in \Sigma^{*} . \Omega^{-1}([t])=\Omega^{-1}(\widehat{\delta}($ Init,$t))=\widehat{\delta}\left(\right.$ Init $\left.^{\prime}, t\right)$. We will now prove the following statements:

$$
\text { (C2) } \Omega^{-1}(\text { Init })=\text { Init }^{\prime}
$$

(C3) $\forall u \in \Sigma^{*} \cdot \forall v \in \Sigma^{*} \cdot \forall \alpha \in \Sigma \cdot[u] \stackrel{\alpha}{\longrightarrow}[v] \Longleftrightarrow \Omega^{-1}([u]) \stackrel{\alpha}{\longrightarrow} \Omega^{-1}([v])$

$$
\text { (C4) } \forall s \in S . s \in F \Longleftrightarrow \Omega^{-1}(s) \in F^{\prime}
$$

$$
\text { (C5) } \forall s \in F \cdot \mu(s)=\mu^{\prime}\left(\Omega^{-1}(s)\right)
$$

First, C2 holds since $\Omega^{-1}($ Init $)=\Omega^{-1}(\widehat{\delta}($ Init,$\epsilon))=\widehat{\delta}\left(\right.$ Init $\left.^{\prime}, \epsilon\right)=$ Init $^{\prime}$. To prove C3, suppose that $[u] \stackrel{\alpha}{\longrightarrow}[v]$. Since $[u]=\widehat{\delta}($ Init,$u)$ we have $[v]=\widehat{\delta}($ Init,$u \bullet \alpha)$. Hence, $\Omega^{-1}([u])=\widehat{\delta}\left(\right.$ Init $\left.^{\prime}, u\right)$ and $\Omega^{-1}([v])=\widehat{\delta}\left(\right.$ Init $\left.^{\prime}, u \bullet \alpha\right)$. But this implies that $\Omega^{-1}([u]) \stackrel{\alpha}{\longrightarrow} \Omega^{-1}([v])$, which proves the forward implication. For the reverse implication suppose that $\Omega^{-1}([u]) \stackrel{\alpha}{\longrightarrow} \Omega^{-1}([v])$. Since $\Omega^{-1}([u])=\widehat{\delta}\left(\right.$ Init $\left.^{\prime}, u\right)$ we again have $\Omega^{-1}([v])=\widehat{\delta}\left(\right.$ Init $\left.^{\prime}, u \bullet \alpha\right)$. Therefore, $[u]=\widehat{\delta}($ Init,$u)$ and $[v]=\widehat{\delta}($ Init,$u \bullet \alpha)$, and hence $[u] \stackrel{\alpha}{\longrightarrow}[v]$.

To prove C4, consider any $s \in S$ such that $s=[u]=\widehat{\delta}($ Init, $u)$. Hence, $\Omega^{-1}(s)=\Omega^{-1}([u])=\widehat{\delta}\left(\right.$ Init $\left.^{\prime}, u\right)$. Then

$$
s \in F \Longleftrightarrow[u] \in F \Longleftrightarrow \exists R \cdot(u, R) \in L \Longleftrightarrow \widehat{\delta}\left(\text { Init }^{\prime}, u\right) \in F^{\prime} \Longleftrightarrow \Omega^{-1}(s) \in F^{\prime}
$$

Finally, we prove C5 by contradiction. Suppose that there exists $s=[u] \in F$ such that $\mu(s) \neq \mu^{\prime}\left(\Omega^{-1}(s)\right)$. Without loss of generality, we can always pick a refusal $R$ such that $\exists R^{\prime} \in \mu(s) \cdot R \subseteq R^{\prime}$ and $\forall R^{\prime} \in \mu^{\prime}\left(\Omega^{-1}(s)\right) \cdot R \nsubseteq R^{\prime}$. Now, we also know that $s=\widehat{\delta}($ Init,$u)$ and $\Omega^{-1}(s)=\widehat{\delta}\left(\right.$ Init $\left.^{\prime}, u\right)$. Therefore, $(u, R) \in \mathcal{L}(A) \backslash \mathcal{L}\left(A^{\prime}\right)$, which implies that $\mathcal{L}(A)=L \neq L=\mathcal{L}\left(A^{\prime}\right)$, a contradiction.

Note that for any $\operatorname{LTS} M, \mathcal{F}(M)$ is regular. ${ }^{5}$ Indeed, the failure automaton corresponding to $M=(S$, Init $, \Sigma, \delta)$ is $A=(M, S, \mu)$ such that $\forall s \in S \cdot \mu(s)=\operatorname{Max}(\{R \mid \operatorname{Ref}(s, R)\})$.

\footnotetext{
5 However, there exist RFLs that do not correspond to any LTS. In particular, any failure language $L$ corresponding to some LTS must satisfy the following condition: $\exists R \subseteq \Sigma \cdot(\epsilon, R) \in L$. Thus, the RFL $\{(\alpha, \emptyset)\}$ does not correspond to any LTS.
} 


\section{$4 \quad$ Assume Guarantee for Deadlock}

We now present an assume-guarantee style [Pnueli 85] proof rule for deadlock detection in systems composed of two components. We use the notion of parallel composition proposed in the theory of CSP [Hoare 85] and define it formally.

Definition 8 (LTS Parallel Composition) Consider LTSs $M_{1}=\left(S_{1}\right.$, Init $\left._{1}, \Sigma_{1}, \delta_{1}\right)$ and $M_{2}=\left(S_{2}\right.$, Init $\left._{2}, \Sigma_{2}, \delta_{2}\right)$. Then the parallel composition of $M_{1}$ and $M_{2}$, denoted by $M_{1} \amalg M_{2}$, is the LTS $\left(S_{1} \times S_{2}\right.$, Init I $_{1} \times$ Init $\left._{2}, \Sigma_{1} \cup \Sigma_{2}, \delta\right)$, such that $\left(\left(s_{1}, s_{2}\right), \alpha,\left(s_{1}^{\prime}, s_{2}^{\prime}\right)\right) \in \delta$ iff the following holds:

$$
\forall i \in\{1,2\} \cdot\left(\alpha \in \Sigma_{i} \wedge\left(s_{i}, \alpha, s_{i}^{\prime}\right) \in \delta_{i}\right) \vee\left(\alpha \notin \Sigma_{i} \wedge s_{i}=s_{i}^{\prime}\right)
$$

Without loss of generality, we assume that both $M_{1}$ and $M_{2}$ have the same alphabet $\Sigma$. Indeed, any system with two components having different alphabets, say $\Sigma_{1}$ and $\Sigma_{2}$, can be converted to a bisimilar (and hence deadlock-equivalent) system [Chaki 05a] with two components, each having the same alphabet $\Sigma_{1} \cup \Sigma_{2}$. Thus, all languages and automata we consider here will also be over the same alphabet $\Sigma$.

We now extend the notion of parallel composition to failure languages. Observe that the composition involves set-intersection on the trace part and set-union on the refusal part of failures. Proofs of all the lemma are detailed in Section 5.

Definition 9 (Failure Language Composition) The parallel composition of any two failure languages $L_{1}$ and $L_{2}$, denoted by $L_{1} \| L_{2}$, is defined as follows:

$$
L_{1} \| L_{2}=\left\{\left(t, R_{1} \cup R_{2}\right) \mid\left(t, R_{1}\right) \in L_{1} \wedge\left(t, R_{2}\right) \in L_{2}\right\}
$$

Lemma 3 For any failure languages $L_{1}, L_{2}, L_{1}^{\prime}$ and $L_{2}^{\prime}$, the following holds:

$$
\left(L_{1} \subseteq L_{1}^{\prime}\right) \wedge\left(L_{2} \subseteq L_{2}^{\prime}\right) \Longrightarrow\left(L_{1} \| L_{2}\right) \subseteq\left(L_{1}^{\prime} \| L_{2}^{\prime}\right)
$$

Proof. Let $(t, R)$ be any failure in $\left(L_{1} \| L_{2}\right)$. Then there exists refusals $R_{1}$ and $R_{2}$ such that: (A) $R=R_{1} \cup R_{2}$, (B) $\left(t, R_{1}\right) \in L_{1}$ and (C) $\left(t, R_{2}\right) \in L_{2}$. From (B), (C), and the premise of the lemma, we have (D) $\left(t, R_{1}\right) \in L_{1}^{\prime}$ and $(\mathbf{E})\left(t, R_{2}\right) \in L_{2}^{\prime}$. But then from $(\mathbf{A}),(\mathbf{D}),(\mathbf{E})$ and Definition 9, we have $(t, R) \in\left(L_{1}^{\prime} \| L_{2}^{\prime}\right)$, which completes the proof.

Definition 10 (FLA Parallel Composition) Consider two $F L A s A_{1}=\left(M_{1}, F_{1}, \mu_{1}\right)$ and $A_{2}=\left(M_{2}, F_{2}, \mu_{2}\right)$. The parallel composition of $A_{1}$ and $A_{2}$, denoted by $A_{1} \amalg A_{2},{ }^{6}$ is defined as the FLA $\left(M_{1} \amalg M_{2}, F_{1} \times F_{2}, \mu\right)$ such that:

$$
\mu\left(s_{1}, s_{2}\right)=\operatorname{Max}\left(\left\{R_{1} \cup R_{2} \mid R_{1} \in \mu_{1}\left(s_{1}\right) \wedge R_{2} \in \mu_{2}\left(s_{2}\right)\right\}\right)
$$

6 We overload the operator $\amalg$ to denote parallel composition in the context of both LTSs and FLAs. The actual meaning of the operator will be clear from its context. 
Let $M_{1}, M_{2}$ be LTSs and $A_{1}, A_{2}$ be FLAs. Then, the following two lemmas bridge the concepts of composition between automata and languages.

Lemma $4 \mathcal{F}\left(M_{1} \amalg M_{2}\right)=\mathcal{F}\left(M_{1}\right) \| \mathcal{F}\left(M_{2}\right)$.

Proof. For any LTSs $M_{1}$ and $M_{2}$ over the same alphabet $\Sigma$, it can be proved that

$$
\mathcal{F}\left(M_{1} \amalg M_{2}\right)=\left\{\left(t, R_{1} \cup R_{2}\right) \mid\left(t, R_{1}\right) \in \mathcal{F}\left(M_{1}\right) \wedge\left(t, R_{2}\right) \in \mathcal{F}\left(M_{2}\right)\right\}
$$

The lemma then follows from the above fact and Definition 9.

Lemma $5 \mathcal{L}\left(A_{1} \amalg A_{2}\right)=\mathcal{L}\left(A_{1}\right) \| \mathcal{L}\left(A_{2}\right)$.

Proof. Let $A_{1}=\left(M_{1}, F_{1}, \mu_{1}\right)$ and $A_{2}=\left(M_{2}, F_{2}, \mu_{2}\right)$ where $M_{1}=\left(S_{1}\right.$, Init $\left._{1}, \Sigma, \delta_{1}\right)$ and $M_{2}=\left(S_{2}\right.$, Init $\left._{2}, \Sigma, \delta_{2}\right)$. Then we know that $A_{1} \amalg A_{2}=\left(M_{1} \amalg M_{2}, F_{1} \times F_{2}, \mu\right)$. Let $(t, R)$ be any element of $\mathcal{L}\left(A_{1} \amalg A_{2}\right)$. Then, we know that

$$
\exists\left(s_{1}, s_{2}\right) \in \widehat{\delta}\left(\text { Init }_{1} \times \text { Init }_{2}, t\right) \cap F_{1} \times F_{2} \cdot \exists R^{\prime} \in \mu\left(s_{1}, s_{2}\right) \cdot R \subseteq R^{\prime}
$$

From the definition of $\mu$, we find that

$$
\exists R_{1} \in \mu_{1}\left(s_{1}\right) \cdot \exists R_{2} \in \mu_{2}\left(s_{2}\right) \cdot R \subseteq R_{1} \cup R_{2}
$$

Therefore, $\left(t, R_{1}\right) \in \mathcal{L}\left(A_{1}\right),\left(t, R_{2}\right) \in \mathcal{L}\left(A_{2}\right)$, and $(t, R) \in \mathcal{L}\left(A_{1}\right) \| \mathcal{L}\left(A_{2}\right)$. This statement proves that $\mathcal{L}\left(A_{1} \amalg A_{2}\right) \subseteq \mathcal{L}\left(A_{1}\right) \| \mathcal{L}\left(A_{2}\right)$. Now, let $(t, R)$ be any element of $\mathcal{L}\left(A_{1}\right) \| \mathcal{L}\left(A_{2}\right)$. Then, we know that

$$
\exists s_{1} \in \widehat{\delta}\left(\text { Init }_{1}, t\right) \cap F_{1} \cdot \exists s_{2} \in \widehat{\delta}\left(\text { Init }_{2}, t\right) \cap F_{2} \cdot \exists R_{1} \in \mu_{1}\left(s_{1}\right) \cdot \exists R_{2} \in \mu_{2}\left(s_{2}\right) \cdot R \subseteq R_{1} \cup R_{2}
$$

Therefore, $\left(s_{1}, s_{2}\right) \in \widehat{\delta}\left(\right.$ Init $_{1} \times$ Init $\left._{2}, t\right) \cap F_{1} \times F_{2}$ and $\exists R^{\prime} \in \mu\left(s_{1}, s_{2}\right) . R \subseteq R^{\prime}$. Hence $(t, R) \in \mathcal{L}\left(A_{1} \amalg A_{2}\right)$. This show that $\mathcal{L}\left(A_{1}\right) \| \mathcal{L}\left(A_{2}\right) \subseteq \mathcal{L}\left(A_{1} \amalg A_{2}\right)$ and completes the proof.

Regular Failure Language Containment (RFLC). We develop a general compositional framework for checking RFLC. This framework is also applicable to deadlock detection since, as we will show later, deadlock freedom is a form of RFLC. Recall that RFLs are not closed under complementation. Given RFLs $L_{1}$ and $L_{2}$, it is not possible to verify $L_{1} \subseteq L_{2}$ in the usual manner by checking if $L_{1} \cap \overline{L_{2}}=\emptyset$. However, as is shown by the following crucial lemma, it is possible to check containment between RFLs using their representations in terms of deterministic FLA without having to complement the automaton that corresponds to $L_{2}$.

Lemma 6 Consider any FLA $A_{1}$ and $A_{2}$. Let $A_{1}^{\prime}=\left(M_{1}, F_{1}, \mu_{1}\right)$ and $A_{2}^{\prime}=\left(M_{2}, F_{2}, \mu_{2}\right)$ be the FLA obtained by determinizing $A_{1}$ and $A_{2}$ respectively, and let $M_{1}=\left(S_{1}\right.$, Init $\left._{1}, \Sigma, \delta_{1}\right)$ and $M_{2}=\left(S_{2}\right.$, Init $\left._{2}, \Sigma, \delta_{2}\right)$. Then $\mathcal{L}\left(A_{1}\right) \subseteq \mathcal{L}\left(A_{2}\right)$ iff for every reachable state $\left(s_{1}, s_{2}\right)$ of $M_{1} \amalg M_{2}$ the following condition holds:

$$
s_{1} \in F_{1} \Longrightarrow\left(s_{2} \in F_{2} \wedge\left(\forall R_{1} \in \mu_{1}\left(s_{1}\right) \cdot \exists R_{2} \in \mu_{2}\left(s_{2}\right) \cdot R_{1} \subseteq R_{2}\right)\right)
$$


Proof. First, we note that $\mathcal{L}\left(A_{1}\right)=\mathcal{L}\left(A_{1}^{\prime}\right)$ and $\mathcal{L}\left(A_{2}\right)=\mathcal{L}\left(A_{2}^{\prime}\right)$. Now let $M_{1}=\left(S_{1}\right.$, Init $\left._{1}, \Sigma, \delta_{1}\right)$ and $M_{2}=\left(S_{2}\right.$, Init $\left._{2}, \Sigma, \delta_{2}\right)$. For the forward implication, we prove the contrapositive. Suppose that there exists a reachable state $\left(s_{1}, s_{2}\right)$ of $M_{1} \amalg M_{2}$ such that $s_{1} \in F_{1}$ and either $s_{2} \notin F_{2}$ or $\exists R_{1} \in \mu_{1}\left(s_{1}\right) \cdot \forall R_{2} \in \mu_{2}\left(s_{2}\right) . R_{1} \nsubseteq R_{2}$. Since $M_{1}$ and $M_{2}$ are deterministic, let $t \in \Sigma^{*}$ be a trace such that $\left(s_{1}, s_{2}\right)=\widehat{\delta}\left(\right.$ Init $_{1} \times$ Init $\left._{2}, t\right)$. Now, we choose a refusal $R$ as follows. If $s_{2} \notin F_{2}$ then let $R$ be any element of $\mu_{1}\left(s_{1}\right)$. Otherwise let $R$ be some $R_{1} \in \mu_{1}\left(s_{1}\right)$ such that $\forall R_{2} \in \mu_{2}\left(s_{2}\right) . R_{1} \not \subset R_{2}$. Now, it follows that $(t, R) \in \mathcal{L}\left(A_{1}^{\prime}\right) \backslash \mathcal{L}\left(A_{2}^{\prime}\right)$. Hence $\mathcal{L}\left(A_{1}^{\prime}\right) \nsubseteq \mathcal{L}\left(A_{2}^{\prime}\right)$ and therefore $\mathcal{L}\left(A_{1}\right) \nsubseteq \mathcal{L}\left(A_{2}\right)$.

For the reverse implication we also prove the contrapositive. Suppose $\mathcal{L}\left(A_{1}\right) \nsubseteq \mathcal{L}\left(A_{2}\right)$ and let $(t, R)$ be any element of $\mathcal{L}\left(A_{1}\right) \backslash \mathcal{L}\left(A_{2}\right)=\mathcal{L}\left(A_{1}^{\prime}\right) \backslash \mathcal{L}\left(A_{2}^{\prime}\right)$. Let $s_{1}=\widehat{\delta}\left(\right.$ Init $\left._{1}, t\right)$ and $s_{2}=\widehat{\delta}\left(\right.$ Init $\left._{2}, t\right)$. But we know that $\exists R_{1} \in \mu_{1}\left(s_{1}\right) \cdot R \subseteq R_{1}$ and either $s_{2} \notin F_{2}$ or $\forall R_{2} \in \mu_{2}\left(s_{2}\right) \cdot R \nsubseteq R_{2}$. However, this implies that $s_{1} \in F_{1}$ and either $s_{2} \notin F_{2}$ or $\exists R_{1} \in \mu_{1}\left(s_{1}\right) \cdot \forall R_{2} \in \mu_{2}\left(s_{2}\right) \cdot R_{1} \not \subset R_{2}$. In addition, $\left(s_{1}, s_{2}\right)$ is a reachable state of $M_{1} \amalg M_{2}$. This completes the proof.

In other words, we can check if $\mathcal{L}\left(A_{1}\right) \subseteq \mathcal{L}\left(A_{2}\right)$ by determinizing $A_{1}$ and $A_{2}$, constructing the product of the underlying LTSs and checking if the condition in Lemma 6 holds on every reachable state of the product. In essence, the condition says that for every reachable state $\left(s_{1}, s_{2}\right)$, if $s_{1}$ is final, then $s_{2}$ is also final and each refusal $R_{1}$ labeling $s_{1}$ is contained in some refusal $R_{2}$ labeling $s_{2}$.

Now suppose that $\mathcal{L}\left(A_{1}\right)$ is obtained by composing two RFLs $L_{1}$ and $L_{2}$, i.e., $\mathcal{L}\left(A_{1}\right)=$ $L_{1} \| L_{2}$ and let $\mathcal{L}\left(A_{2}\right)=L_{S}$, the specification language. To check RFLC between $L_{1} \| L_{2}$ and $L_{S}$, the approach presented in Lemma 6 requires us to directly compose $L_{1}, L_{2}$ and $L_{S}$, a potentially expensive computation. In the following, we first show that checking deadlock-freedom is a particular case of RFLC and then present a compositional technique to check RFLC (and hence deadlock-freedom) that avoids composing $L_{1}$ and $L_{2}$ (or their FLA representations) directly.

Deadlock as RFLC. Given three RFLs $L_{1}, L_{2}$ and $L_{S}$, we can use our regular language containment algorithm to verify whether $\left(L_{1} \| L_{2}\right) \subseteq L_{S}$. If this is the case, then our algorithm returns TRUE. Otherwise it returns FALSE along with a counterexample $C E \in\left(L_{1} \| L_{2}\right) \backslash L_{S}$. Also, we assume that $L_{1}, L_{2}$ and $L_{S}$ are represented as FLA. To use our algorithm for deadlock detection, recall that for any two LTSs $M_{1}$ and $M_{2}, M_{1} \amalg M_{2}$ is deadlock free iff $\mathcal{F}\left(M_{1} \amalg M_{2}\right) \subseteq \overline{L_{D l k}}$. Let $L_{1}=\mathcal{F}\left(M_{1}\right), L_{2}=\mathcal{F}\left(M_{2}\right)$ and $L_{S}=\overline{L_{D l k}}$. Using Lemma 4 , this deadlock check reduces to verifying if $L_{1} \| L_{2} \subseteq L_{S}$. Observe that we can use our RFLC algorithm provided $L_{1}, L_{2}$ and $L_{S}$ are regular. Recall that since $M_{1}$ and $M_{2}$ are LTSs, $L_{1}$ and $L_{2}$ are regular. Also, $\overline{L_{D l k}}$ is regular, since it is accepted by the failure automaton $A=(M, F, \mu)$ such that: (i) $M=(\{s\},\{s\}, \Sigma, \delta)$, (ii) $\delta=\{s \stackrel{\alpha}{\longrightarrow} s \mid \alpha \in \Sigma\}$, $F=\{s\}$, and (iv) $\mu(s)=\operatorname{Max}(\{R \mid R \subset \Sigma\})$. For instance, if $\Sigma=\{a, b, c\}$, then $\mu(s)=\{\{a, b\},\{b, c\},\{c, a\}\}$. Thus, we find that deadlock detection is just a specific instance of RFLC.

Suppose we are given three RFLs $L_{1}, L_{2}$ and $L_{S}$ in the form of their accepting FLA $A_{1}, A_{2}$ and $A_{S}$. To check $L_{1} \| L_{2} \subseteq L_{S}$, we can construct the FLA $A_{1} \amalg A_{2}$ (see Lemma 10) and then check if $\mathcal{L}\left(A_{1} \amalg A_{2}\right) \subseteq \mathcal{L}\left(A_{S}\right)$ (see Lemma 5 and 6). The problem with this naive approach is statespace explosion. To alleviate this problem, we present a compositional language containment scheme based on AG-style reasoning in the next section. 


\subsection{A Non-Circular AG Rule}

Consider RFLs $L_{1}, L_{2}$ and $L_{S}$. We are interested in checking whether $L_{1} \| L_{2} \subseteq L_{S}$. In this context the following non-circular AG proof rule, called AG-NC, is both sound and complete:

$$
\frac{L_{1} \| L_{A} \subseteq L_{S} \quad L_{2} \subseteq L_{A}}{L_{1} \| L_{2} \subseteq L_{S}}
$$

Proof. The completeness of AG-NC follows from the fact that if the conclusion holds, then $L_{2}$ can be used as $L_{A}$ to discharge the two premises. To prove soundness, let us assume that the two premises hold. Then from the second premise and Lemma 3, we have $L_{1}\left\|L_{2} \subseteq L_{1}\right\| L_{A}$. Combining this statement with the first premise, we get $L_{1} \| L_{2} \subseteq L_{S}$ which is the desired conclusion.

In principle, AG-NC enables us to prove $L_{1} \| L_{2} \subseteq L_{S}$ by discovering an assumption $L_{A}$ that discharges its two premises. In practice, we are left with two critical problems. First, it provides no effective method for constructing an appropriate assumption $L_{A}$. Second, if no appropriate assumption exists; that is, if the conclusion of AG-NC does not hold, then AG-NC does not help in obtaining a counterexample to $L_{1} \| L_{2} \subseteq L_{S}$. In this report we develop and employ a learning algorithm that solves both the above problems. Specifically, our algorithm learns automatically and incrementally the weakest assumption $L_{W}$ that can discharge the first premise of AG-NC. During this process, it is guaranteed to reach one of the following two situations in a finite number of steps and to terminate with the correct result:

1. It discovers an assumption that can discharge both premises of AG-NC and terminates with TRUE.

2. It discovers a counterexample $C E$ to $L_{1} \| L_{2} \subseteq L_{S}$ and returns FALSE along with $C E$.

We present complete details of our algorithm, as well as its complexity, later in Section 5 . First we discuss formally the notion of the weakest assumption $L_{W}$.

\subsection{Weakest Assumption}

Consider the proof rule AG-NC. For any $L_{1}$ and $L_{S}$, let $\widehat{L}$ be the set of all languages that can discharge the first premise of AG-NC. In other words, $\widehat{L}=\left\{L_{A} \mid\left(L_{1} \| L_{A}\right) \subseteq L_{S}\right\}$. The following central theorem asserts that $\widehat{L}$ contains a unique weakest (maximal) element $L_{W}$ that is also regular. This result is crucial for showing the termination of our approach.

Theorem 1 Let $L_{1}$ and $L_{S}$ be any RFLs and $f$ is a failure. Let us define a language $L_{W}$ as follows: $L_{W}=\left\{f \mid\left(L_{1} \|\{f\}\right) \subseteq L_{S}\right\}$. Then the following holds: (i) $L_{1} \| L_{W} \subseteq L_{S}$, (ii) $\forall L . L_{1} \| L \subseteq L_{S} \Longleftrightarrow L \subseteq L_{W}$, and (iii) $L_{W}$ is regular. 
Proof. We first prove (i) by contradiction. Suppose there exists $\left(t, R_{1}\right) \in L_{1}$ and $\left(t, R_{2}\right) \in L_{W}$ such that $\left(t, R_{1} \cup R_{2}\right) \notin L_{S}$. But then $\left(t, R_{1} \cup R_{2}\right) \in L_{1} \|\left\{\left(t, R_{2}\right)\right\}$ which means $L_{1} \|\left\{\left(t, R_{2}\right)\right\} \nsubseteq L_{S}$. However, this contradicts $\left(t, R_{2}\right) \in L_{W}$.

Now, we only prove the forward implication of (ii). The reverse implication follows from (i) and Lemma 3. This proof is also by contradiction. Suppose there exists a language $L$ such that $L_{1} \| L \subseteq L_{S}$ and $L \nsubseteq L_{W}$. Then there exists some $\left(t, R_{2}\right) \in L \backslash L_{W}$. But since $\left(t, R_{2}\right) \notin L_{W}$, there exists $\left(t, R_{1}\right) \in L_{1}$ such that $\left(t, R_{1} \cup R_{2}\right) \notin L_{S}$. However, this means that $\left(t, R_{1} \cup R_{2}\right) \in L_{1} \| L$, which contradicts $L_{1} \| L \subseteq L_{S}$.

Finally, to prove that $L_{W}$ is regular we construct an FLA $A_{W}$ such that $\mathcal{L}\left(A_{W}\right)=L_{W}$. Let $A_{1}=\left(M_{1}, F_{1}, \mu_{1}\right)$ and $A_{S}=\left(M_{S}, F_{S}, \mu_{S}\right)$ be deterministic and complete FLA accepting $L_{1}$ and $L_{S}$ respectively such that $M_{1}=\left(S_{1}\right.$, Init $\left._{1}, \Sigma, \delta_{1}\right)$ and $M_{S}=\left(S_{S}\right.$, Init $\left._{S}, \Sigma, \delta_{S}\right)$. Then $A_{W}=\left(M_{1} \amalg M_{S}, F_{W}, \mu_{W}\right)$. To define the set of final states $F_{W}$ and the labeling function $\mu_{W}$ of $A_{W}$, we define the extended labeling function $\widehat{\mu}: S \rightarrow 2^{\widehat{\Sigma}}$ of any FLA as follows:

$\widehat{\mu}(s)=\mu(s)$ if $s$ is a final state and $\emptyset$ otherwise. Then the extended labeling function $\widehat{\mu}$ of $A_{W}$ is defined as follows:

$$
\widehat{\mu}\left(s_{1}, s_{S}\right)=\left\{R \in \widehat{\Sigma} \mid \forall R_{1} \in \widehat{\mu}\left(s_{1}\right) \cdot \exists R_{S} \in \widehat{\mu}\left(s_{S}\right) \cdot\left(R_{1} \cup R\right) \subseteq R_{S}\right\}
$$

Note that the set $\widehat{\mu}\left(s_{1}, s_{S}\right)$ is always downward-closed. In other words

$$
\forall R \in \widehat{\Sigma} \cdot \forall R^{\prime} \in \widehat{\Sigma} \cdot R \in \widehat{\mu}\left(s_{1}, s_{S}\right) \wedge R^{\prime} \subseteq R \Longrightarrow R^{\prime} \in \widehat{\mu}\left(s_{1}, s_{S}\right)
$$

Then the definitions of $F_{W}$ and $\mu_{W}$ follow naturally as below:

$$
\begin{gathered}
F_{W}=\left\{\left(s_{1}, s_{S}\right) \mid \widehat{\mu}\left(s_{1}, s_{S}\right) \neq \emptyset\right\} \\
\forall\left(s_{1}, s_{S}\right) \in F_{W} \cdot \mu_{W}\left(s_{1}, s_{S}\right)=\operatorname{Max}\left(\widehat{\mu}\left(s_{1}, s_{S}\right)\right)
\end{gathered}
$$

Note that since $A_{1}$ and $A_{S}$ are both deterministic and complete, so is $A_{W}$. Also, for any state $\left(s_{1}, s_{S}\right)$ of $A_{W}$ and any $t \in \Sigma^{*}$, we have $\widehat{\delta}\left(\left(s_{1}, s_{S}\right), t\right)=\left(\widehat{\delta}\left(s_{1}, t\right), \widehat{\delta}\left(s_{S}, t\right)\right)$. We now prove that $\mathcal{L}\left(A_{W}\right)=L_{W}$. Consider any failure $(t, R) \in\left(\Sigma^{*} \times \widehat{\Sigma}\right)$. Let $\left(s_{1}, s_{S}\right)=\widehat{\delta}\left(\left(\right.\right.$ Init $_{1}$, Init $\left.\left._{S}\right), t\right)$. We consider two sub-cases.

Case $1\left[(t, R) \in \mathcal{L}\left(A_{W}\right)\right]$. Then we know that $R \in \widehat{\mu}\left(s_{1}, s_{S}\right)$. Now consider the language $L=L_{1} \|\{(t, R)\}$. By Definition 9, any element of $L$ must be of the form $\left(t, R_{1} \cup R\right)$ for some $R_{1} \in \widehat{\mu}\left(s_{1}\right)$. Also, from the definition of $\widehat{\mu}$ above we have $\exists R_{S} \in \widehat{\mu}\left(s_{S}\right) \cdot\left(R_{1} \cup R\right) \subseteq R_{S}$. Hence $\left(t, R_{1} \cup R\right) \in L_{S}$. Since $\left(t, R_{1} \cup R\right)$ is an arbitrary element of $L$ we conclude that $L \subseteq L_{S}$. Hence, from the definition of $L_{W}$ above we have $(t, R) \in L_{W}$ which completes the proof of this subcase.

Case $2\left[(t, R) \notin \mathcal{L}\left(A_{W}\right)\right]$. In this case, $R \notin \widehat{\mu}\left(s_{1}, s_{S}\right)$. Then, from the definition of $\widehat{\mu}$ above, we have $\exists R_{1} \in \widehat{\mu}\left(s_{1}\right) \cdot \forall R_{S} \in \widehat{\mu}\left(s_{S}\right) \cdot\left(R_{1} \cup R\right) \nsubseteq R_{S}$. Now consider the language $L=L_{1} \|\{(t, R)\}$. By Definition $9,\left(t, R_{1} \cup R\right) \in L$. However, from $\forall R_{S} \in \widehat{\mu}\left(s_{S}\right) \cdot\left(R_{1} \cup R\right) \nsubseteq R_{S}$, we have $\left(t, R_{1} \cup R\right) \notin L_{S}$. Hence, $L \nsubseteq L_{S}$. Thus, from the definition of $L_{W}$ above we have $(t, R) \notin L_{W}$, which completes the proof of this subcase and of the entire theorem.

Now that we have proved that the weakest environment assumption $L_{W}$ is regular, we can apply a learning algorithm to iteratively construct an FLA assumption that accepts $L_{W}$. In 
particular, we develop a learning algorithm $L^{F}$ that iteratively learns the minimal DFLA corresponding to $L_{W} . L^{F}$ asks queries about $L_{W}$ to a minimally adequate teacher (MAT) and learns from the answers. In the next Section, we present $L^{F}$. Subsequently, in section 6 , we describe how $L^{F}$ is used in our compositional language containment procedure. If you are only interested in the overall compositional deadlock-detection algorithm and not the intricacies of $L^{F}$, you should skip to Section 6 . 


\section{$5 \quad$ Learning FLA}

In this section we present an algorithm $L^{F}$ to learn the minimal FLA that accepts an unknown RFL $U$. Our algorithm will use a minimally adequate teacher (MAT) that can answer two kinds of queries regarding $U$ :

1. membership query: Given a failure $e$, the MAT returns TRUE if $e \in U$ and FALSE otherwise.

2. candidate query: Given a DFLA $C$, the MAT returns TRUE if $\mathcal{L}(C)=U$. Otherwise, it returns FALSE along with a counterexample failure $C E \in(\mathcal{L}(C) \backslash U) \bigcup(U \backslash \mathcal{L}(C))$.

\subsection{Observation Table}

$L^{F}$ uses an observation table to record the information it obtains by querying the MAT. The rows and columns of the table correspond to specific traces and failures respectively. Formally, a table is a triple $\left(\mathbb{T}, \mathbb{E}, \mathbb{R}\right.$ ) where: (i) $\mathbb{T} \subseteq \Sigma^{*}$ is a set of traces; (ii) $\mathbb{E} \subseteq \Sigma^{*} \times \widehat{\Sigma}$ is a set of failures or experiments; and (iii) $\mathbb{R}$ is a function from $\widehat{\mathbb{T}} \times \mathbb{E}$ to $\{0,1\}$ where $\widehat{\mathbb{T}}=\mathbb{T} \cup(\mathbb{T} \bullet \Sigma)$. For any table $\mathcal{T}=(\mathbb{T}, \mathbb{E}, \mathbb{R})$, the function $\mathbb{R}$ is defined as follows:

$$
\forall t \in \widehat{\mathbb{T}} \cdot \forall e=\left(t^{\prime}, R\right) \in \mathbb{E} \cdot \mathbb{R}(t, e)=1 \Longleftrightarrow\left(t \bullet t^{\prime}, R\right) \in U
$$

Thus, given $\mathbb{T}$ and $\mathbb{E}$, algorithm $L^{F}$ can compute $\mathbb{R}$ via membership queries to the MAT. For any $t \in \widehat{\mathbb{T}}$, we write $\mathbb{R}(t)$ to mean the function from $\mathbb{E}$ to $\{0,1\}$ defined as follows:

$$
\forall e \in \mathbb{E} \cdot \mathbb{R}(t)(e)=\mathbb{R}(t, e)
$$

An observation table $\mathcal{T}=(\mathbb{T}, \mathbb{E}, \mathbb{R})$ is said to be well-formed iff the following holds:

$$
\forall t_{1} \in \mathbb{T} \cdot \forall t_{2} \in \mathbb{T} \cdot t_{1} \neq t_{2} \Longrightarrow \mathbb{R}\left(t_{1}\right) \neq \mathbb{R}\left(t_{2}\right)
$$

Essentially, this means that any two distinct rows $t_{1}$ and $t_{2}$ of a well-formed table can be distinguished by some experiment $e \in \mathbb{E}$. There is also an upper bound on the number of rows of any well-formed table, as expressed by the following lemma.

Lemma 7 Let $n$ be the number of states of the minimal DFLA accepting $U$ and let $\mathcal{T}=(\mathbb{T}, \mathbb{E}, \mathbb{R})$ be any well-formed observation table. Then $|\mathbb{T}| \leq n$.

Proof. The proof is by contradiction. Suppose that $|\mathbb{T}|>n$. Let the minimal DFLA accepting $U$ be $A$. Then there exists two distinct traces $t_{1}$ and $t_{2}$ in $\mathbb{T}$ such that $\widehat{\delta}\left(\right.$ Init,$\left.t_{1}\right)=\widehat{\delta}\left(\right.$ Init,$\left.t_{2}\right)$. In other words, the FLA $A$ reaches the same state on input $t_{1}$ and $t_{2}$. But since $\mathcal{T}$ is well-formed, there exists some failure $e=(t, p) \in \mathbb{E}$ such that $\mathbb{R}\left(t_{1}, e\right) \neq \mathbb{R}\left(t_{2}, e\right)$. In other words, $\left(t_{1} \bullet t, p\right) \in U$ iff $\left(t_{2} \bullet t, p\right) \notin U$. This case is impossible, since $A$ would reach the same state on inputs $t_{1} \bullet t$ and $t_{2} \bullet t$. 


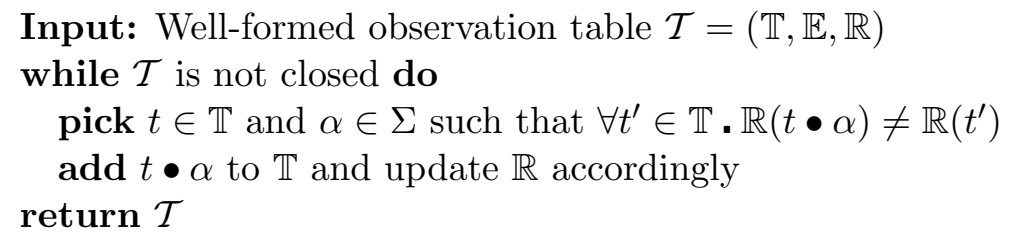

Figure 2: Algorithm MakeClosed.

Closed Observation Table. An observation table $\mathcal{T}=(\mathbb{T}, \mathbb{E}, \mathbb{R})$ is said to be closed iff it satisfies the following:

$$
\forall t \in \mathbb{T} \cdot \forall \alpha \in \Sigma \cdot \exists t^{\prime} \in \mathbb{T} \cdot \mathbb{R}(t \bullet \alpha)=\mathbb{R}\left(t^{\prime}\right)
$$

Intuitively, if we extend any trace $t \in \mathbb{T}$ by any action $\alpha$, then the result is indistinguishable from an existing trace $t^{\prime} \in \mathbb{T}$ under the current set of experiments $\mathbb{E}$. Note that any well-formed table can be extended so that it is both well-formed and closed. This extension can be achieved by the algorithm MakeClosed shown in Figure 2. Observe that at every step of MakeClosed, the table $\mathcal{T}$ remains well-formed and hence, by Lemma 7, cannot grow infinitely. Also note that restricting the occurrence of refusals to $\mathbb{E}$ allows us to avoid considering the exponential possible refusal extensions of a trace while closing the table. Exponential number of membership queries are required only if all possible refusals occur in $\mathbb{E}$.

\subsection{Overall $L^{F}$ Algorithm}

Algorithm $L^{F}$ is iterative. It initially starts with a table $\mathcal{T}=(\mathbb{T}, \mathbb{E}, \mathbb{R})$ such that $\mathbb{T}=\{\epsilon\}$ and $\mathbb{E}=\emptyset$. The initial table is well-formed. Subsequently, in each iteration $L^{F}$ performs the following steps:

1. Make $\mathcal{T}$ closed by invoking MakeClosed.

2. Construct candidate DFLA $C$ from $\mathcal{T}$ and make candidate query with $C$.

3. If the answer is TRUE, $L^{F}$ terminates with $C$ as the final answer.

4. Otherwise, $L^{F}$ uses the counterexample $C E$ to the candidate query to add a single new failure to $\mathbb{E}$ and repeats from Step 1 .

In each iteration, $L^{F}$ either terminates with the correct answer (Step 3) or adds a new failure to $\mathbb{E}$ (Step 4). In the latter scenario, the new failure to be added is constructed so that it guarantees an upper bound on the total number of iterations of $L^{F}$. This construction ensures its ultimate termination. We now present the procedures for: (i) constructing a candidate DFLA $C$ from a closed and well-formed table $\mathcal{T}$ (used in Step 2 above), and (ii) adding a new failure to $\mathbb{E}$ based on a counterexample to a candidate query (Step 4). 


\subsection{Candidate Construction}

Let $\mathcal{T}=(\mathbb{T}, \mathbb{E}, \mathbb{R})$ be a closed and well-formed observation table. The candidate DFLA $C$ is constructed from $\mathcal{T}$ as follows: $C=(M, F, \mu)$ and $M=(S$, Init, $\Sigma, \delta)$ such that

- $S=\mathbb{T}:$ Each state of $M$ corresponds to a distinct row of $\mathcal{T}$.

- Init $=\epsilon$ : The initial state of $M$ corresponds to the empty trace. The empty trace always belongs to $\mathbb{T}$, since initially $\mathbb{T}=\{\epsilon\}$ and subsequently $\mathbb{T}$ grows monotonically.

- $\delta$ is constructed as follows: Consider any $t \in \mathbb{T}$ and $\alpha \in \Sigma$. Since $\mathcal{T}$ is well-formed and closed, we know that there exists an unique $t^{\prime} \in \mathbb{T}$ such that $\mathbb{R}(t \bullet \alpha)=\mathbb{R}\left(t^{\prime}\right)$. Then, we add $t \stackrel{\alpha}{\longrightarrow} t^{\prime}$ to $\delta$. In other words

$$
\delta=\left\{t \stackrel{\alpha}{\longrightarrow} t^{\prime} \mid \mathbb{R}(t \bullet \alpha)=\mathbb{R}\left(t^{\prime}\right)\right\}
$$

- The state corresponding to a row $t$ is final if there exists a successful failure $e \in \mathbb{E}$ from $t$ such that the trace component of $e$ is empty. In other words

$$
F=\{t \mid \exists e=(\epsilon, p) \in \mathbb{E} \cdot \mathbb{R}(t, e)=1\}
$$

Finally, the mapping $\mu$ is constructed as follows. Let $t \in F$ be any final state of $M$. Consider the set $P=\{R \mid e=(\epsilon, R) \in \mathbb{E} \wedge \mathbb{R}(t, e)=1\}$. From the definition of $F$ above, we know that $P \neq \emptyset$. Then $\mu(t)=\operatorname{Max}(P)$. We now present the algorithm to add new failures to $\mathcal{T}$ using a counterexample $C E$ to a candidate query made with a DFLA $C$ constructed as above.

\subsection{Adding New Failures}

Let $C=(M, F, \mu)$ be a candidate DFLA such that $M=(S$, Init, $\Sigma, \delta)$. Let $C E=(t, R)$ be a counterexample to a candidate query made with $C$. In other words,

$C E \in \mathcal{L}(C) \Longleftrightarrow C E \notin U$. The algorithm NewExp adds a single new failure to $\mathcal{T}$ as follows. Let $t=\alpha_{1} \bullet \ldots \bullet \alpha_{k}$. For $0 \leq i \leq k$, let $t_{i}$ be the prefix of $t$ of length $i$ and $t^{i}$ be the suffix of $t$ of length $k-i$. In other words, for $0 \leq i \leq k$, we have $t_{i} \bullet t^{i}=t$.

Additionally, for $0 \leq i \leq k$, let $s_{i}$ be the state of $C$ reached by executing $t_{i}$. In other words, $s_{i}=\widehat{\delta}\left(t_{i}\right)$. Since the candidate $C$ was constructed from an observation table $\mathcal{T}$, it corresponds to a row of $\mathcal{T}$, which in turn corresponds to a trace. Let us also refer to this trace as $s_{i}$. Finally, let $b_{i}=1$ if the failure $\left(s_{i} \bullet t^{i}, R\right) \in U$ and 0 otherwise. Note that we can compute $b_{i}$ by evaluating $s_{i}$ and then making a membership query with $\left(s_{i} \bullet t^{i}, R\right)$. In particular, $s_{0}=\epsilon$, and hence $b_{0}=1$ if $C E \in U$ and 0 otherwise. We now consider two cases.

Case 1: $\left[b_{0}=0\right]$ means that $C E \notin U$ and hence $C E \in \mathcal{L}(C)$. Recall that $C E=(t, R)$ and $t=\alpha_{1} \bullet \ldots \bullet \alpha_{k}$. Consider the state $s_{k}=\widehat{\delta}(t)$ as described above. Since $C E \in \mathcal{L}(C)$, we know that $s_{k} \in F$ and $\exists R^{\prime} \in \mu\left(s_{k}\right) . R \subseteq R^{\prime}$.

Also, since $C$ was constructed (see Section 5.3) from a table $\mathcal{T}=(\mathbb{T}, \mathbb{E}, \mathbb{R})$, we know that $\left(\epsilon, R^{\prime}\right) \in \mathbb{E}$ and $\mathbb{R}\left(s_{k}, R^{\prime}\right)=1$. However, this means that the failure $\left(s_{k}, R^{\prime}\right) \in U$. Since 
$R \subseteq R^{\prime}$, it follows that $\left(s_{k}, R\right) \in U$ and therefore $b_{k}=1$. Since $b_{0}=0$ and $b_{k}=1$, there exists an index $j \in\{0, \ldots, k\}$ such that $b_{j}=0$ and $b_{j+1}=1$. In this case, $L^{F}$ finds such an index $j$ and adds the failure $\left(t^{j+1}, R\right)$ to $\mathbb{E}$. We now show that the failure $e=\left(t^{j+1}, R\right)$ has a special property.

Since $C$ contained a transition $s_{j} \stackrel{\alpha_{j+1}}{\longrightarrow} s_{j+1}$, it must be the case that $\mathbb{R}\left(s_{j} \bullet \alpha_{j+1}\right)=\mathbb{R}\left(s_{j+1}\right)$. However, $\mathbb{R}\left(s_{j} \bullet \alpha_{j+1}, e\right)=b_{j} \neq b_{j+1}=\mathbb{R}\left(s_{j+1}, e\right)$. Thus, after adding $e$ to $\mathbb{E}$, the table is no longer closed. Hence, when $L^{F}$ attempts to make $\mathcal{T}$ closed in the very next iteration, it will be forced to increase the number of rows of $\mathcal{T}$ by at least one.

Case 2: $\left[b_{0}=1\right]$ means that $C E \in U$ and hence $C E \notin \mathcal{L}(C)$. We consider two subcases. First, suppose that $b_{k}=0$. Then there exists an index $j \in\{0, \ldots, k\}$ such that $b_{j}=1$ and $b_{j+1}=0$. In this case, $L^{F}$ finds such an index $j$ and adds the failure $\left(t^{j+1}, R\right)$ to $\mathbb{E}$. As in Case 1 above, this guarantees that the number of rows of $\mathcal{T}$ must strictly increase in the next iteration of $L^{F}$.

Otherwise, we have $b_{k}=1$, but this means that the failure $\left(s_{k}, R\right) \in U$. However, since $C E \notin \mathcal{L}(C)$ we know that either $s_{k}$ is not a final state of $C$ or $\forall R^{\prime} \in \mu\left(s_{k}\right) \cdot R \nsubseteq R^{\prime}$. In this scenario, $L^{F}$ computes a maximal element $R_{\max }$ such that $R \subseteq R_{\max }$ and $\left(s_{k}, R_{\max }\right) \in U$. It then adds the failure $\left(\epsilon, R_{\max }\right)$ to $\mathbb{E}$.

The addition of $\left(\epsilon, R_{\max }\right)$ to $\mathbb{E}$ must lead to at least one of two consequences in the next iteration of $L^{F}$ in terms of the next computed candidate DFLA. First, the number of rows of $\mathcal{T}$ and states of the candidate may increase. Otherwise, either the state $s_{k}$ changes from a non-final to a final state or the set $\mu\left(s_{k}\right)$ gets an additional element, namely, $R_{\max }$.

Relationship Between $L^{F}$ and $L^{*}$. Although $L^{F}$ and $L^{*}$ are similar in their overall structure, there are a number of differences. First, since $L^{F}$ learns a failure automaton, the columns of the observation table store failures instead of traces as in $L^{*}$. Second, when $L^{F}$ learns from a counterexample, every iteration may not involve an increase in the number of states; instead, the failure label on one or more states may be enlarged.

\subsection{Correctness of $L^{F}$}

Algorithm $L^{F}$ always returns the correct answer in Step 3, since it always does so after a successful candidate query. To confirm that $L^{F}$ always terminates, observe that in every iteration, the candidate $C$ that $L^{F}$ computes undergoes at least one of these three changes:

- (Ch1) The number of states of $C$ and the number of rows in the observation table $\mathcal{T}$, increases.

- (Ch2) The states and transitions of $C$ remain unchanged, but a state of $C$ that was previously non-final becomes final.

- (Ch3) The states, transitions and final states of $C$ remain unchanged, but for some final states $s$ of $C$, the size of $\mu(s)$ increases.

Of the above changes, Ch1 can happen at most $n$ times where $n$ is the number of states of the minimal DFLA accepting $U$. Between any two consecutive occurrences of $\mathbf{C h 1}$, there can 
only be a finite number of occurrences of $\mathbf{C h} \mathbf{2}$ and $\mathbf{C h} 3$. Hence, there can only be a finite number of iterations of $L^{F}$. Therefore, $L^{F}$ always terminates.

Number of Iterations. To analyze the complexity of $L^{F}$, we must impose a tighter bound on the number of iterations. We already know that Ch1 can happen at most $n$ times. Since a final state can never become non-final, Ch2 can also occur at most $n$ times. Now, let the minimal DFLA accepting $U$ be $A=(M, F, \mu)$ such that $M=(S$, Init, $\Sigma, \delta)$. Consider the set $P=\bigcup_{s \in F} \mu(s)$ and let $n^{\prime}=|P|$. Since each Ch3 adds an element to $\mu(s)$ for some $s \in F$, the total number of occurrences of $\mathbf{C h} \mathbf{3}$ is at most $n^{\prime}$. Therefore, the maximum number of iterations of $L^{F}$ is $2 n+n^{\prime}=\mathcal{O}\left(n+n^{\prime}\right)$.

Time Complexity. Let us make the standard assumption that each MAT query takes $\mathcal{O}(1)$ time. From the above discussion, we see that the number of columns of the observation table is at most $\mathcal{O}\left(n+n^{\prime}\right)$. The number of rows is at most $\mathcal{O}(n)$. Let us assume that the size of $\Sigma$ is a constant. Then, the number of membership queries, and hence time, needed to fill up the table is $\mathcal{O}\left(n\left(n+n^{\prime}\right)\right)$.

Let $m$ be the length of the longest counterexample returned by a candidate query. Then to add each new failure, we have to make $\mathcal{O}(\log (m))$ membership queries to find the appropriate index $j$. Also, let the time required to find the maximal element $R_{\max }$ be $\mathcal{O}\left(m^{\prime}\right)$. The total time required for constructing each new failure is $\mathcal{O}\left(\left(n+n^{\prime}\right)\left(\log (m)+m^{\prime}\right)\right)$. Finally, the number of candidate queries equals the number of iterations and hence is $\mathcal{O}\left(n+n^{\prime}\right)$. In summary, we find that the time complexity of $L^{F}$ is $\mathcal{O}\left(\left(n+n^{\prime}\right)\left(n+\log (m)+m^{\prime}\right)\right)$, which is polynomial in $n, n^{\prime}, m$ and $m^{\prime}$.

Space Complexity. Let us again make the standard assumption that each MAT query takes $\mathcal{O}(1)$ space. Since the queries are made sequentially, the total space requirement for all of them is still $\mathcal{O}(1)$. Also, the procedure for constructing a new failure can be performed in $\mathcal{O}(1)$ space. A trace corresponding to a table row can be $\mathcal{O}(n)$ long, and there are $\mathcal{O}(n)$ of them. A failure corresponding to a table column can be $\mathcal{O}(m)$ long, and there are $\mathcal{O}\left(n+n^{\prime}\right)$ of them. Space required to store the table elements is $\mathcal{O}\left(n\left(n+n^{\prime}\right)\right)$. Hence the total space required for the observation table is $\mathcal{O}\left((n+m)\left(n+n^{\prime}\right)\right)$. The space required to store computed candidates is $\mathcal{O}\left(n^{2}\right)$. Therefore, the total space complexity is $\mathcal{O}\left((n+m)\left(n+n^{\prime}\right)\right)$, which is also polynomial in $n, n^{\prime}$ and $m$. 


\section{Compositional Language Containment}

Given RFLs $L_{1}, L_{2}$ and $L_{S}$ (in the form of FLA that accept them), we want to check whether $L_{1} \| L_{2} \subseteq L_{S}$. If not, we also want to generate a counterexamples $C E \in\left(L_{1} \| L_{2}\right) \backslash L_{S}$. To this end, we invoke the $L^{F}$ algorithm to learn the weakest environment corresponding to $L_{1}$ and $L_{S}$. We present an implementation strategy for the MAT to answer the membership and candidate queries that $L^{F}$ poses. In the following, we assume that $A_{1}, A_{2}$ and $A_{S}$ are the given FLAs such that $\mathcal{L}\left(A_{1}\right)=L_{1}, \mathcal{L}\left(A_{2}\right)=L_{2}$ and $\mathcal{L}\left(A_{S}\right)=L_{S}$.

Membership Query. The answer to a membership query with failure $e=(t, R)$ is TRUE if the following condition (which can be effectively decided) holds or is otherwise FALSE: $\forall\left(t, R_{1}\right) \in L_{1} \cdot\left(t, R_{1} \cup R\right) \in L_{S}$.

Candidate Query. A candidate query with an FLA $C$ is answered step-wise as follows:

1. Check if $\mathcal{L}\left(A_{1} \amalg C\right) \subseteq \mathcal{L}\left(A_{S}\right)$. If not, let $\left(t, R_{1} \cup R\right)$ be the counterexample obtained. Note that $(t, R) \in \mathcal{L}(C) \backslash U$. We return FALSE to $L^{F}$ along with the counterexample $(t, R)$. If $\mathcal{L}\left(A_{1} \amalg C\right) \subseteq \mathcal{L}\left(A_{S}\right)$, we proceed to Step 2 .

2. Check if $\mathcal{L}\left(A_{2}\right) \subseteq \mathcal{L}(C)$. If so, we have obtained an assumption, namely, $\mathcal{L}(C)$, that discharges both premises of AG-NC. In this case, the overall language containment algorithm terminates with TRUE. Otherwise, let $\left(t^{\prime}, R^{\prime}\right)$ be the counterexample obtained. We proceed to Step 3.

3. We check if there exists $\left(t^{\prime}, R_{1}^{\prime}\right) \in \mathcal{L}\left(A_{1}\right)$ such that $\left(t^{\prime}, R_{1}^{\prime} \cup R^{\prime}\right) \notin \mathcal{L}\left(A_{S}\right)$. If so, then $\left(t^{\prime}, R_{1}^{\prime} \cup R^{\prime}\right) \in \mathcal{L}\left(A_{1} \amalg A_{2}\right) \backslash \mathcal{L}\left(A_{S}\right)$, and the overall language containment algorithm terminates with FALSE and the counterexample $\left(t^{\prime}, R_{1}^{\prime} \cup R^{\prime}\right)$. Otherwise, $\left(t^{\prime}, R^{\prime}\right) \in U \backslash \mathcal{L}(C)$, and we return FALSE to $L^{F}$ along with the counterexample $\left(t^{\prime}, R^{\prime}\right)$.

Note that in these queries, we are never required to compose $A_{1}$ with $A_{2}$. In practice, the candidate $C$ (that we compose with $A_{1}$ in Step 1 of the candidate query) is much smaller than $A_{2}$. Thus, we are able to alleviate the statespace explosion problem. Also, note that our procedure will ultimately terminate with the correct result from either Step 2 or 3 of the candidate query. This assumption follows from the correctness of $L^{F}$ algorithm: In the worst case, the candidate query will be made with an FLA $C$ such that $\mathcal{L}(C)=L_{W}$. In this scenario, termination is guaranteed to occur due to Theorem 1. 


\section{$7 \quad$ Arbitrary Components and Circularity}

We investigated two approaches for handling more than two components. First, we applied AG-NC recursively. This approach can be demonstrated for languages $L_{1}, L_{2}, L_{3}$ and $L_{S}$ by the following proof-rule.

$$
\frac{L_{1} \| L_{A}^{1} \subseteq L_{S} \quad \frac{L_{2} \| L_{A}^{2} \subseteq L_{A}^{1} \quad L_{3} \subseteq L_{A}^{2}}{L_{2} \| L_{3} \subseteq L_{A}^{1}}}{L_{1}\left\|L_{2}\right\| L_{3} \subseteq L_{S}}
$$

At the top level, we apply AG-NC on the two languages $L_{1}$ and $L_{2} \| L_{3}$. Now the second premise becomes $L_{2} \| L_{3} \subseteq L_{A}^{1}$, and we can again apply AG-NC. In terms of the implementation of the MAT, the only difference is in Step 2 of the candidate query (see Section 6). More specifically, we now invoke the language containment procedure recursively with $\mathcal{L}\left(A_{2}\right), \mathcal{L}\left(A_{3}\right)$ and $\mathcal{L}(C)$ instead of checking directly for $\mathcal{L}\left(A_{2}\right) \subseteq \mathcal{L}(C)$. This technique can be extended to any finite number of components.

Circular AG Rule. We also explored a circular AG rule. Unlike AG-NC however, the circular rule is specific to deadlock detection and not applicable to language containment in general. For any RFL $L$, let us write $W(L)$ to denote the weakest assumption against which $L$ does not deadlock. In other words, $\forall L^{\prime} . L \| L^{\prime} \subseteq \overline{L_{D l k}} \Longleftrightarrow L^{\prime} \subseteq W(L)$. It can be shown that: (PROP) $\forall t \in \Sigma^{*} \cdot \forall R \in \widehat{\Sigma} \cdot(t, R) \in L \Longleftrightarrow(t, \Sigma \backslash R) \notin W(L)$. The following theorem provides a circular AG rule for deadlock detection.

Theorem 2 Consider any two RFLs $L_{1}$ and $L_{2}$. Then the following proof rule, which we call AG-Circ, is both sound and complete.

$\frac{L_{1} \| L_{A}^{1} \subseteq \overline{L_{D l k}} \quad \begin{array}{l}L_{2} \| L_{A}^{2} \subseteq \overline{L_{D l k}} \\ W\left(L_{A}^{1}\right) \| W\left(L_{A}^{2}\right) \subseteq \overline{L_{D l k}}\end{array}}{L_{1} \| L_{2} \subseteq \overline{L_{D l k}}}$

Proof. We first prove soundness by contradiction. Assume that three premises hold but the conclusion does not. There exists a trace $t$ and a refusal $R$ such that $(t, R) \in L_{1}$ and $(t, \Sigma \backslash R) \in L_{2}$. From the first premise, we see that $(t, \Sigma \backslash R) \notin L_{A}^{1}$. Similarly, from the second premise, we get $(t, R) \notin L_{A}^{2}$. Therefore, we have $(t, R) \in W\left(L_{A}^{1}\right)$ and $(t, \Sigma \backslash R) \in W\left(L_{A}^{2}\right)$. But then $(t, \Sigma) \in W\left(L_{A}^{1}\right) \| W\left(L_{A}^{2}\right)$, which contradicts the third premise.

We now prove completeness. Let us assume the conclusion. We show that if we set $L_{A}^{1}=W\left(L_{1}\right)$ and $L_{A}^{2}=W\left(L_{2}\right)$, then all three premises are satisfied. The first two premises follow from the definitions of $W\left(L_{1}\right)$ and $W\left(L_{2}\right)$. We prove the third premise by contradiction. Suppose there exists a trace $t$ and a refusal $R$ such that $(t, R) \in W\left(W\left(L_{1}\right)\right)$ and $(t, \Sigma \backslash R) \in W\left(W\left(L_{2}\right)\right)$, but then we know that $(t, \Sigma \backslash R) \notin W\left(L_{1}\right)$ and $(t, R) \notin W\left(L_{2}\right)$. 
However, this supposition means that $(t, R) \in L_{1}$ and $(t, \Sigma \backslash R) \in L_{2}$ and implies that $(t, \Sigma) \in L_{1} \| L_{2}$, which contradicts the conclusion.

Implementation. To use this rule for deadlock detection of two components $L_{1}$ and $L_{2}$ (the approach generalizes to any finite number of components), we use this iterative procedure:

1. Using the first premise, construct a candidate $C_{1}$ similar to Step 1 of the candidate query in AG-NC (see Section 6). Similarly, using the second premise, construct another candidate $C_{2}$. Construction of $C_{1}$ and $C_{2}$ proceeds exactly as in the case of AG-NC.

2. Check if $W\left(\mathcal{L}\left(C_{1}\right)\right) \| W\left(\mathcal{L}\left(C_{2}\right)\right) \subseteq \overline{L_{D l k}}$. This check is done either directly or via a compositional language containment using AG-NC. We compute the automata for $W\left(\mathcal{L}\left(C_{1}\right)\right)$ and $W\left(\mathcal{L}\left(C_{2}\right)\right)$ using the procedure described in the proof of Theorem 1. If the check succeeds, then there is no deadlock in $L_{1} \| L_{2}$ and we exit successfully. Otherwise, we proceed to Step 3.

3. From the counterexample obtained above, construct $t \in \Sigma^{*}$ and $R \in \widehat{\Sigma}$ such that $(t, R) \in W\left(\mathcal{L}\left(C_{1}\right)\right)$ and $(t, \Sigma \backslash R) \in W\left(\mathcal{L}\left(C_{2}\right)\right)$. Check if $(t, R) \in L_{1}$ and $(t, \Sigma \backslash R) \in L_{2}$. If both these checks pass, then we have a counterexample $t$ to the overall deadlock-detection problem and we terminate unsuccessfully. Otherwise, without loss of generality, suppose $(t, R) \notin L_{1}$. But then, from PROP,$(t, \Sigma \backslash R) \in W\left(L_{1}\right)$. Again from PROP, since $(t, R) \in W\left(\mathcal{L}\left(C_{1}\right)\right),(t, \Sigma \backslash R) \notin \mathcal{L}\left(C_{1}\right)$, which is equivalent to a failed candidate query for $C_{1}$ with counterexample $(t, \Sigma \backslash R)$. We repeat from Step 1 above.

Note that even though we have presented AG-Circ in the context of only two components, it generalizes to an arbitrary, but finite, number of components. 


\section{Experimental Validation}

We implemented our algorithms in the ComFoRT [Chaki 05b] reasoning framework and experimented with a set of real-life examples. All our experiments were done on a $2.4 \mathrm{GHz}$ Pentium 4 machine running RedHat Linux 9 and with a time limit of one hour and a memory limit of $2 \mathrm{~GB}$. Our results are summarized in Table 3. The $M C$ benchmarks are derived from Micro-C Version 2.70, a lightweight OS for real-time embedded applications. The IPC benchmark is based on an interprocess communication library used by an industrial robot controller software. The ide, syn, $m x$ and $t g 3$ examples are based on Linux device drivers. Finally, $D P$ is a synthetic benchmark based on the well-known dining philosophers example.

For each example, we obtained a set of benchmarks by increasing the number of components. For each such benchmark, we tested one version without deadlock and another with an artificially introduced deadlock. In all cases, deadlock was caused by incorrect synchronization between components - the only difference was in the synchronization mechanism. Specifically, the dining philosophers synchronized using "forks." In all other examples, synchronization was achieved via a shared "lock."

For each benchmark, a finite LTS model was constructed via a predicate abstraction [Chaki 05b] that transformed the synchronization behavior into appropriate actions. For example, in the case of the ide benchmark, calls to the spin_lock and spin_unlock functions were transformed into lock and unlock actions, respectively. These function calls make sense because, for instance, multiple threads executing the driver for a specific device will acquire and release a common lock specific to that device by invoking spin_lock and spin_unlock respectively.

For each abstraction, appropriate predicates were supplied externally so that the resulting models would be precise enough to display the presence or absence of deadlock. In addition, care was taken to ensure that the abstractions were sound with respect to deadlocks, that is, the extra behavior introduced did not eliminate any deadlock in the concrete system. Each benchmark was verified using explicit brute-force statespace exploration (referred to in Table 3 as "Plain"), the non-circular AG rule (referred to as AG-NC), and the circular AG rule (referred to as AG-Circ). When using AG-Circ (i.e., checking if $\left.W\left(\mathcal{L}\left(C_{1}\right)\right) \| W\left(\mathcal{L}\left(C_{2}\right)\right) \subseteq \overline{L_{D l k}}\right)$, Step 2 was done via compositional language containment using AG-NC.

We observe that the AG-based methods outperform the naive approach for most benchmarks. More importantly, for each benchmark, the growth in memory consumption combined with the increasing number of components is benign for both AG-based approaches. This bounded growth indicates that AG reasoning is effective in combating statespace explosion even for deadlock detection. We also note that larger assumptions (and hence time and memory) are required for detecting deadlocks as opposed to detecting deadlock freedom. Among the AG-based approaches, AG-Circ is generally faster than AG-NC, but it consumed negligible extra memory on a few occasions. In several cases, AG-NC runs out of time, while AG-Circ is able to terminate successfully. Overall, whenever AG-NC and AG-Circ differ significantly in any real-life example, AG-Circ is superior. 

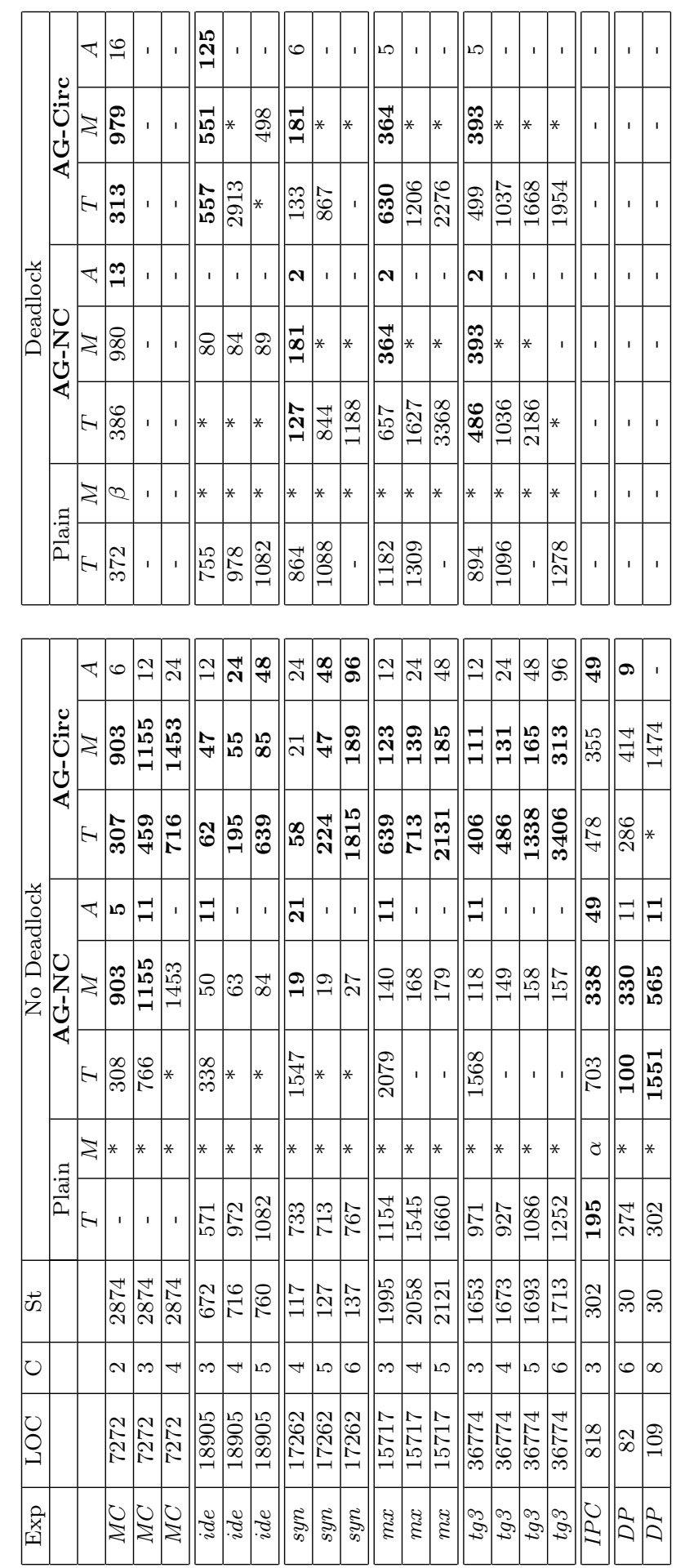

Figure 3: $\quad$ Experimental results. $C=\#$ of components; $S t=\#$ of states of largest component; $T=$ time (seconds); $M=$ memory (MB); $A=$ \# of states of largest assumption; * = resource exhaustion; - = data unavailable; $\alpha=1247 ; \beta=1708$. Best figures are shown in bold. 


\section{Conclusion}

In this report, we have extended the learning-based automated assume-guarantee paradigm to deadlock detection. We have defined a new kind of automata, which are similar to finite automata but accept failures instead of traces. We have also developed an algorithm called $L^{F}$ that is similar to $L^{*}$ and learns the minimal failure automata accepting an unknown regular failure language using a minimally adequate teacher. We have shown how $L^{F}$ can be used for compositional deadlock detection using both circular and non-circular assume-guarantee rules. Finally, we have implemented our technique and obtained encouraging experimental results on several nontrivial benchmarks.

We believe this work leaves several avenues open for further investigation. An intriguing question concerns the relationship between learning and abstraction refinement. While both approaches construct approximations iteratively, abstraction refinement always strengthens its approximation. On the other hand, learning may either strengthen or weaken its assumption depending on the counterexample to the candidate query that the MAT returns. Another issue is the possibility of increasing the efficiency of our approach via symbolic implementations. Finally, the question of extending the automated assume-guarantee via learning paradigm to yet other types of conformances is not yet settled. For instance, it is unclear how you may use this paradigm to carry out model checking against specifications written in a temporal logic, such as the $\mu$-calculus, CTL or LTL. 


\section{References}

[Alur 05a]

[Alur 05b]

[Amla 03]

[Angluin 87]

[Barringer 03]

[Brookes 91]

[Chaki 05a]
Alur, R.; Cerny, P.; Gupta, G.; Madhusudan, P.; Nam, W.; \& Srivastava, A. "Synthesis of Interface Specifications for Java Classes," 98-109. Edited by Palsberg, J. \& Abadi, M.

Symposium on Principles of Programming Languages 2005

(Popl 05). Long Beach, CA, January 12-14, 2005. New York, NY: ACM Press, January 2005.

Alur, R.; Madhusudan, P.; \& Nam, W. "Symbolic Compositional Verification by Learning Assumptions," 548-562. Edited by Etessami, K. \& Rajamani, S. K. Proceedings of the 17th International Conference on Computer Aided Verification (CAV'05), Volume 3576 of Lecture Notes in Computer Science. Edinburgh, Scotland, July 6-10, 2005. New York, NY: Springer-Verlag, July 2005.

Amla, N.; Emerson, E. A.; Namjoshi, K. S.; \& Trefler, R. J. "Abstract Patterns of Compositional Reasoning," 423-438. Edited by Amadio, R. M. \& Lugiez, D. Proceedings of the 14 th International Conference on Concurrency Theory (CONCUR '03), Volume 2761 of Lecture Notes in Computer Science. Marseille, France, September 3-5, 2003. New York, NY: Springer-Verlag, September 2003.

Angluin, D. "Learning Regular Sets from Queries and Counterexamples." Information and Computation 75, 2 (November 1987): 87-106.

Barringer, H.; Giannakopoulou, D.; \& Păsăreanu, C. S. "Proof Rules for Automated Compositional Verification," 14-21. Proceedings of the 2nd Workshop on Specification and Verification of Component Based Systems (SAVCBS '03). Helsinki, Finland, September 1-2, 2003. Ames, Iowa: Iowa State University, September 2003.

Brookes, S. D. \& Roscoe, A. W. "Deadlock Analysis of Networks of Communicating Processes." Distributed Computing 4 (December 1991): 209-230.

Chaki, S.; Clarke, E. M.; Sinha, N.; \& Thati, P. "Automated Assume-Guarantee Reasoning for Simulation Conformance," 534-547. Edited by Etessami, K. \& Rajamani, S. K. Proceedings of the 17th International Conference on Computer Aided Verification (CAV '05), Volume 3576 of Lecture Notes in Computer Science. Edinburgh, Scotland, July 6-10, 2005. New York, NY: Springer-Verlag, July 2005. 
[Chaki 05b]

[Cobleigh 03]

[de Roever 98]

[Ernst 99]

[Fournet 04]
Chaki, S.; Ivers, J.; Sharygina, N.; \& Wallnau, K. "The ComFoRT Reasoning Framework," 164-169. Edited by Etessami, K. \& Rajamani, S. K. Proceedings of the 17th International Conference on Computer Aided Verification (CAV '05), Volume 3576 of Lecture Notes in Computer Science. Edinburgh, Scotland, July 6-10, 2005. New York, NY: Springer-Verlag, July 2005.

Cobleigh, J. M.; Giannakopoulou, D.; \& Păsăreanu, C. S. "Learning Assumptions for Compositional Verification," 331-346. Edited by Garavel, H. \& Hatcliff, J. Proceedings of the 9th International Conference on Tools and Algorithms for the Construction and Analysis of Systems (TACAS '03), Volume 2619 of Lecture Notes in Computer Science. Warsaw, Poland, April 7-11, 2003. New York, NY: Springer-Verlag, April 2003.

de Roever, W. P.; Langmaack, H.; \& Pnueli, A., editors. Compositionality: The Significant Difference, International Symposium, COMPOS'97, Revised Lectures, Volume 1536 of Lecture Notes in Computer Science, Bad Malente, Germany, September 8-12, 1997. New York, NY: Springer-Verlag, 1998.

Ernst, M. D.; Cockrell, J.; Griswold, W. G.; \& Notkin, D. "Dynamically Discovering Likely Program Invariants to Support Program Evolution," 213-224. Proceedings of the 21st International Conference on Software Engineering (ICSE '99), Los Angeles, CA, May 16-22, 1999. Los Angeles, CA: IEEE Computer Society Press, May 1999.

Fournet, C.; Hoare, C. A. R.; Rajamani, S. K.; \& Rehof, J. "Stuck-Free Conformance," 242-254. Edited by Alur, R. \& Peled, D. Proceedings of the 16th International Conference on Computer Aided Verification (CAV'04), Volume 3114 of Lecture Notes in Computer Science, Boston, MA, July 13-17, 2004. New York, NY: Springer-Verlag, July 2004.

[Giannakopoulou 02] Giannakopoulou, D.; Păsăreanu, C. S.; \& Barringer, H. "Assumption Generation for Software Component Verification," 3-12. Proceedings of the 17th International Conference on Automated Software Engineering (ASE '02). Edinburgh, Scotland, September 23-27, 2002. Los Alamitos, CA: IEEE Computer Society Press, September 2002.

[Groce 02] Groce, A.; Peled, D.; \& Yannakakis, M. "Adaptive Model Checking," 357-370. Edited by Katoen, J. P. \& Stevens, P. Proceedings of the Eighth International Conference on Tools and Algorithms for the Construction and Analysis of Systems (TACAS '02), Volume 2280 of Lecture Notes in Computer 
[Grumberg 94]

[Habermehl 05]

[Hoare 85]

[Holzmann 03]

[Kahlon 05]

[Kozen 01]

[McMillan 97]

[Overkamp 97]

[Peled 99]
Science. Grenoble, France, April 8-12, 2002. New York, NY:

Springer-Verlag, April 2002.

Grumberg, O. \& Long, D. E. "Model Checking and Modular Verification." ACM Transactions on Programming Languages and System (TOPLAS) 16, 3 (May 1994): 843-871.

Habermehl, P. \& Vojnar, T. "Regular Model Checking Using Inference of Regular Languages," 21-36. Proceedings of the 6th International Workshop on Verification of Infinite-State Systems (INFINITY '04), London, England, September 4, 2004, Volume 138(3) of Electronic Notes in Theoretical Computer Science. December 2005.

Hoare, C. A. R. Communicating Sequential Processes. London, England: Prentice Hall, 1985.

Holzmann, G. The SPIN Model Checker: Primer and Reference Manual. Boston, MA: Addison-Wesley, 2003.

Kahlon, V.; Ivancic, F.; \& Gupta, A. "Reasoning About Threads Communicating via Locks," 505-518. Edited by Etessami, K. \& Rajamani, S. K. Proceedings of the 17th International Conference on Computer Aided Verification (CAV '05), Volume 3576 of Lecture Notes in Computer Science. Edinburgh, Scotland, July 6-10, 2005. New York, NY: Springer-Verlag, July 2005.

Kozen, D. Automata on Guarded Strings and Applications (Technical Report TR2001-1833). Ithaca, NY: Cornell University, 2001.

McMillan, K. L. "A Compositional Rule for Hardware Design Refinement," 24-35. Edited by Grumberg, O. Proceedings of the 9th International Conference on Computer Aided Verification (CAV'97), Volume 1254 of Lecture Notes in Computer Science. Haifa, Israel, June 22-27, 1997. New York, NY: Springer-Verlag, June 1997.

Overkamp, A. "Supervisory Control Using Failure Semantics and Partial Specifications." IEEE Transactions on Automatic Control 42, 4 (April 1997): 498-510.

Peled, D.; Vardi, M. Y.; \& Yannakakis, M. "Black Box Checking," 225-240. Edited by Wu, J.; Chanson, S. T.; \& Gao, Q. Proceedings of the Joint International Conference on Formal Description Techniques for Distributed Systems and Communication Protocols (FORTE '99), Volume 156 of IFIP Conference Proceedings, Beijing, China, October 5-8, 1999. Norwell, MA: Kluwer Academic Publishers, October 1999. 
[Pnueli 85]

[Rivest 93]

[Roscoe 97]

[Williams 05]
Pnueli, A. "In Transition from Global to Modular Temporal Reasoning About Programs." Logics and Models of Concurrent Systems 13 (1985): 123-144.

Rivest, R. L. \& Schapire, R. E. "Inference of Finite Automata Using Homing Sequences." Information and Computation 103, 2 (April 1993): 299-347.

Roscoe, A. W. The Theory and Practice of Concurrency. New York, NY: Prentice-Hall International, 1997.

Williams, A.; Thies, W.; \& Ernst, M. D. "Static Deadlock Detection for Java Libraries," 602-629. Edited by Black, A. P. Proceedings of the 19th European Conference on Object-Oriented Programming (ECOOP '05), Volume 3586 of Lecture Notes in Computer Science. Glasgow, UK, July 25-29, 2005. New York, NY: Springer-Verlag, July 2005. 
SOFTWARE ENGINEERING INSTITUTE 


\begin{tabular}{|c|c|c|c|}
\hline \multicolumn{3}{|c|}{ REPORT DOCUMENTATION PAGE } & $\begin{array}{l}\text { Form Approved } \\
\text { OMB No 0704-0188 }\end{array}$ \\
\hline \multicolumn{4}{|c|}{ 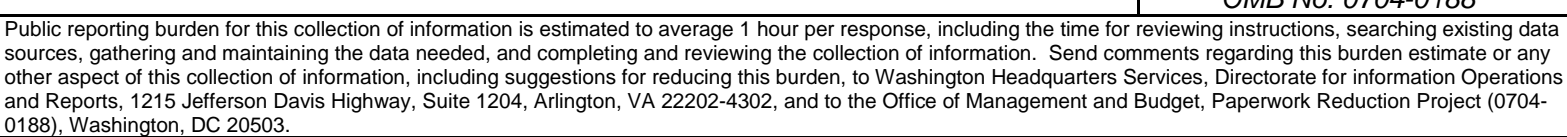 } \\
\hline $\begin{array}{l}\text { 1. } \\
\text { AGENCY USE ONLY } \\
\text { (Leave Blank) }\end{array}$ & \multicolumn{2}{|c|}{\begin{tabular}{|l|l|} 
2. & REPORT DATE \\
& September 2006 \\
\end{tabular}} & $\begin{array}{ll}\text { 3. } & \text { REPORT TYPE AND DATES COVERED } \\
& \text { Final }\end{array}$ \\
\hline \multicolumn{3}{|c|}{$\begin{array}{l}\text { TITLE AND SUBTITLE } \\
\text { Assume-Guarantee Reasoning for Deadlock }\end{array}$} & $\begin{array}{ll}5 . & \text { FUNDING NUMBERS } \\
& \text { FA8721-05-C-0003 } \\
\end{array}$ \\
\hline \multicolumn{4}{|c|}{$\begin{array}{ll}\text { 6. } & \text { AUTHOR(s) } \\
& \text { Sagar Chaki and Nishant Sinha }\end{array}$} \\
\hline \multicolumn{3}{|c|}{$\begin{array}{l}\text { 7. PERFORMING ORGANIZATION NAME(S) AND ADDRESS(ES) } \\
\text { Software Engineering Institute } \\
\text { Carnegie Mellon University } \\
\text { Pittsburgh, PA } 15213\end{array}$} & $\begin{array}{ll}8 . & \text { PERFORMING ORGANIZATION } \\
\text { REPORT NUMBER } \\
\text { CMU/SEI-2006-TN-028 }\end{array}$ \\
\hline \multicolumn{3}{|c|}{$\begin{array}{l}\text { 9. SPONSORING/MONITORING AGENCY NAME(S) AND ADDRESS(ES) } \\
\text { HQ ESC/XPK } \\
5 \text { Eglin Street } \\
\text { Hanscom AFB, MA 01731-2116 }\end{array}$} & $\begin{array}{l}\text { 10. SPONSORING/MONITORING AGENCY } \\
\text { REPORT NUMBER }\end{array}$ \\
\hline \multicolumn{4}{|c|}{ 11. SUPPLEMENTARY NOTES } \\
\hline \multicolumn{3}{|c|}{$\begin{array}{l}\text { 12A DISTRIBUTION/AVALALBILITY STATEMENT } \\
\text { Unclassified/Unlimited, DTIC, NTIS }\end{array}$} & 12B DISTRIBUTION CODE \\
\hline \multicolumn{4}{|c|}{$\begin{array}{l}\text { 13. ABSTRACT (MAXIMUM } 200 \text { wORDS) } \\
\text { The use of learning to automate assume-guarantee style reasoning has received a lot of attention in recent years. This } \\
\text { paradigm has already been used successfully for checking trace containment, as well as simulation between concurrent } \\
\text { systems and their specifications. In this report, the learning-based automated assume-guarantee paradigm is extended } \\
\text { to perform compositional deadlock detection. Failure automata is defined as a generalization of finite automata that } \\
\text { accept regular failure sets. A learning algorithm } \mathrm{LF} \text { is developed that constructs the minimal deterministic failure } \\
\text { automata accepting any unknown regular failure set using a minimally adequate teacher. This report shows how } \mathrm{LF} \text { can } \\
\text { be used for compositional regular failure language containment and deadlock detection, using non-circular and circular } \\
\text { assume-guarantee rules. Finally, an implementation of techniques and encouraging experimental results on several } \\
\text { nontrivial benchmarks are presented. }\end{array}$} \\
\hline \multicolumn{3}{|c|}{$\begin{array}{l}\text { 14. SUBJECT TERMS } \\
\text { deadlock detection, learning algorithm, assume guarantee, failure automata, finite } \\
\text { automata }\end{array}$} & $\begin{array}{l}\text { 15. NUMBER OF PAGES } \\
38\end{array}$ \\
\hline \multicolumn{4}{|l|}{ 16. PRICE CODE } \\
\hline $\begin{array}{l}\text { 17. SECURITY CLASSIFICATION OF } \\
\text { REPORT } \\
\text { Unclassified }\end{array}$ & $\begin{array}{l}\text { 18. SECURITY CLASSIFICATION OF } \\
\text { THIS PAGE } \\
\text { Unclassified }\end{array}$ & $\begin{array}{l}\text { 19. SECURITY CLASSIFICATION OF } \\
\text { ABSTRACT } \\
\text { Unclassified }\end{array}$ & $\begin{array}{l}\text { 20. LIMITATION OF ABSTRACT } \\
\text { UL }\end{array}$ \\
\hline
\end{tabular}

